\title{
ACYCLIC CURVES AND GROUP ACTIONS ON AFFINE TORIC SURFACES
}

\author{
IVAN ARZHANTSEV AND MIKHAIL ZAIDENBERG
}

To Masayoshi Miyanishi on occasion of his 70th birthday

\begin{abstract}
We show that every irreducible, simply connected curve on a toric affine surface $X$ over $\mathbb{C}$ is an orbit closure of a $\mathbb{G}_{m}$-action on $X$. It follows that up to the action of the automorphism group $\operatorname{Aut}(X)$ there are only finitely many non-equivalent embeddings of the affine line $\mathbb{A}^{1}$ in $X$. A similar description is given for simply connected curves in the quotients of the affine plane by small finite linear groups. We provide also an analog of the Jung-van der Kulk theorem for affine toric surfaces, and apply this to study actions of algebraic groups on such surfaces.
\end{abstract}

\section{Contents}

Introduction

1. Preliminaries 3

1.1. Simply connected plane affine curves 3

1.2. The automorphism group of the affine plane 3

2. Subgroups of de Jonquères group and stabilizers of plane curves 5

2.1. Subgroups of the de Jonquères group 5

2.2. Stabilizers of acyclic plane curves 8

3. Acyclic curves on affine toric surfaces 12

3.1. Acyclic curves in the smooth locus 12

3.2. Acyclic curves through the singular point 14

3.3. Acyclic curves as orbit closures 16

3.4. Reducible acyclic curves on affine toric surfaces 17

4. Automorphism groups of affine toric surfaces 19

4.1. Free amalgamated product structure 19

4.2. Algebraic groups actions on affine toric surfaces 24

5. Acyclic curves and automorphism groups of non-toric quotient surfaces 25

References

2010 Mathematics Subject Classification. Primary 14H45, 14M25; Secondary 14H50, 14R20.

Key words and phrases. Affine surface, acyclic curve, automorphism group, torus action, quotient.

This work was done during a stay of the first author at the Institut Fourier, Grenoble and of the second author at the Max Planck Institute of Mathematics, Bonn. We thank both institutions for generous support and hospitality. The work of the first author was supported by a Simons Award. 


\section{INTRODUCTION}

The geometry of affine toric surfaces still attracts the researches ${ }^{1}$. Every affine toric surface over $\mathbb{C}$ except for $\mathbb{A}_{*}^{1} \times \mathbb{A}_{*}^{1}$ and $\mathbb{A}^{1} \times \mathbb{A}_{*}^{1}$, where $\mathbb{A}_{*}^{1}=\mathbb{A}^{1} \backslash\{0\}$, is the quotient of $\mathbb{A}^{2}$ by a small finite cyclic subgroup $G \subseteq \mathrm{GL}(2, \mathbb{C}) .^{2}$

Throughout the paper, by 'acyclic curve' we mean a connected and simply connected complex affine algebraic curve. A classification of acyclic curves on the affine plane, both irreducible and reducible, up to the action of the automorphism group of the plane is well known, see e.g. [1], [37], [47], [53], [55]; we recall it in subsection 1.1 below. In section 3 we classify acyclic curves on affine toric surfaces. Similarly as in [54], actions of one-parameter groups play a crucial role in this classification.

Let $\pi: \mathbb{A}^{2} \rightarrow X=\mathbb{A}^{2} / G$ be the quotient morphism, and let $\mathcal{C}$ be an irreducible acyclic curve on $X$. Then $\pi^{*}(\mathrm{C})$ is an acyclic (reducible and non-reduced, in general) curve on $\mathbb{A}^{2}$. We show (see theorems 3.2 and 3.5) that applying an appropriate automorphism of the affine plane we can transform the curve $\pi^{*}(\mathcal{C})$ and the $G$-action on $\mathbb{A}^{2}$ to canonical forms simultaneously.

In subsection 2.2, given an acyclic plane curve $C$, we describe the stabilizer subgroup $\operatorname{Stab}(C) \subseteq \operatorname{Aut}\left(\mathbb{A}^{2}\right)$ of all automorphisms which preserve $C$. We use this description in subsections 3.1 and 3.2 in order to obtain the canonical forms of irreducible acyclic curves on an affine toric surface $X$. We treat separately the cases of the curves passing or do not passing through the singular point of $X$. This leads in subsection 3.3 to the conclusion that every irreducible acyclic curve on $X$ is the closure of a non-closed orbit of a $\mathbb{G}_{m}$-action on $X$. Furthermore, if such a curve is contained in the smooth locus $X_{\text {reg }}$ then it is smooth and is as well an orbit of a $\mathbb{G}_{a}$-action on $X$, hence is included in a one-parameter family of such curves. We show that any affine toric surface $X$ possesses only finitely many equivalence classes of embedded affine lines (see theorem 3.9). This is an analog of the celebrated Abhyankar-MohSuzuki Embedding Theorem, which says that there is just one class of embeddings of the affine line in the affine plane (see theorem 1.1 below).

The above description enables us to classify in subsection 3.4 all reduced simply connected curves on an affine toric surface, whenever they are irreducible or not.

Section 4 is devoted to the automorphism groups of affine toric surfaces. In theorem 4.2 we obtain an analog of the classical Jung-van der Kulk theorem on a free amalgamated product structure on the group Aut $\left(\mathbb{A}^{2}\right)$ (see theorem 1.4). Using this theorem we describe in theorems 4.15 and 4.17 (reductive) algebraic groups acting effectively on affine toric surfaces.

In the final section 5 we deal with acyclic curves on a quotient $X=\mathbb{A}^{2} / G$ of the affine plane $\mathbb{A}^{2}$ by a nonabelian small finite group $G$. It turns out that the only irreducible acyclic curves on $X$ are the images of the affine lines in $\mathbb{A}^{2}$ passing through the origin (see theorem 5.1). In particular, every such curve is the closure of a $\mathbb{G}_{m}$-orbit and passes through the singular point. Since the family of these curves is preserved under automorphisms, the automorphism group $\operatorname{Aut}(X)$ is rather poor. Namely, it coincides with $N(G) / G$, where $N(G)$ stands for the normalizer of $G$ in $\mathrm{GL}(2, \mathbb{C}$ ) (see theorem 5.3). Consequently, $N(G)$ coincides with the normalizer of $G$ in the full automorphism group Aut $\left(\mathbb{A}^{2}\right)$. As an example, we describe explicitly the affine lines on the quaternion surface $X=\mathbb{A}^{2} / Q_{8}$.

\footnotetext{
${ }^{1}$ See, for instance, the recent paper [20] on the Hilbert scheme of zero-cycles on such a surface.

${ }^{2}$ A finite linear group is small if it does not contain pseudoreflections.
} 
When the paper was finished, S. Kaliman kindly informed us that he also came, for different purposes, to similar conclusions, but never wrote them down. The authors thank S. Kaliman for this information and interesting discussions.

\section{Preliminaries}

In this section we gather some well known facts on the geometry of the affine plane $\mathbb{A}^{2}$ over $\mathbb{C}$ that we need in the sequel.

1.1. Simply connected plane affine curves. By a curve we mean (for short) a complex affine algebraic curve. A curve $C$ is called acyclic if $\pi_{0}(C)=\pi_{1}(C)=1$, i.e. $C$ is connected and simply connected. Two plane curves $C$ and $C^{\prime}$ will be called equivalent if $C^{\prime}=\gamma(C)$ for some $\gamma \in \operatorname{Aut}\left(\mathbb{A}^{2}\right)$. The following theorems provide canonical forms of acyclic and, more generally, of simply connected plane curves.

Theorem 1.1. (Abhankar-Moh [1], Suzuki [47]) Any reduced, irreducible, smooth, acyclic plane curve is equivalent to the affine line $C_{y}=\{y=0\}$. Furthermore, if $C$ is parameterized via a map $\varphi: \mathbb{A}^{1} \stackrel{\simeq}{\longrightarrow} C, \varphi(t)=(p(t), q(t))$, where $p, q \in \mathbb{C}[t]$, then either $\operatorname{deg} p \mid \operatorname{deg} q$ or $\operatorname{deg} q \mid \operatorname{deg} p$.

Using Suzuki's formula for the Euler characteristics of the fibers in a fibration on a smooth affine surface [47] (see also e.g. [19]) it is not difficult to deduce the following corollary.

Corollary 1.2. Any disconnected, simply connected, reduced plane curve is equivalent to a union of $r \geq 2$ parallel lines.

Theorem 1.3. (Lin-Zaidenberg [55])

(a) Any reduced, irreducible, singular, acyclic plane curve is equivalent to one and only one of the curves $C_{a, b}=\left\{y^{a}-x^{b}=0\right\}$, where $1<a<b$ and $\operatorname{gcd}(a, b)=1$.

(b) Any reduced, simply connected plane curve is equivalent to a curve given by one of the equations

$$
y^{\varepsilon_{y}} p(x)=0
$$

or

$$
x^{\varepsilon_{x}} y^{\varepsilon_{y}} \prod_{i=1}^{r}\left(y^{a}-\kappa_{i} x^{b}\right)=0,
$$

where $\varepsilon_{x}, \varepsilon_{y} \in\{0,1\}, p \in \mathbb{C}[t]$ is a polynomial with simple roots, $a, b \geq 1$ and $\operatorname{gcd}(a, b)=1, r>0$, and $\kappa_{i} \in \mathbb{C}^{\times}, i=1, \ldots, r$, are pairwise distinct.

1.2. The automorphism group of the affine plane. In this subsection the base field $\mathbb{k}$ can be arbitrary. We let $\mathbb{A}_{\mathrm{k}}^{n}$ denote the affine $n$-space over $\mathbb{k}$ and $\operatorname{Aff}\left(\mathbb{A}_{\mathrm{k}}^{n}\right)$ the group of all affine transformations of this space. By $\mathrm{JONQ}^{+}\left(\mathbb{A}_{\mathbb{k}}^{2}\right)\left(\mathrm{JONQ}^{-}\left(\mathbb{A}_{\mathbb{k}}^{2}\right)\right.$, respectively) we denote the group of the de Jonqières transformations

$$
\Phi^{+}:(x, y) \longmapsto(\alpha x+f(y), \beta y+\gamma),
$$

respectively,

$$
\Phi^{-}:(x, y) \longmapsto(\alpha x+\gamma, \beta y+f(x)),
$$


where $\alpha, \beta \in \mathbb{k}^{\times}, \gamma \in \mathbb{k}$, and $f \in \mathbb{k}[t]$. The subgroup

$$
\operatorname{Aff}{ }^{ \pm}\left(\mathbb{A}_{\mathbb{k}}^{2}\right)=\operatorname{Aff}\left(\mathbb{A}_{\mathbb{k}}^{2}\right) \cap \operatorname{JONQ}^{ \pm}\left(\mathbb{A}_{\mathbb{k}}^{2}\right)
$$

consists of all upper (lower, respectively) triangular affine transformations $\Phi^{ \pm}$with $\operatorname{deg} f \leq 1$. The structure of the automorphism group $\operatorname{Aut}\left(\mathbb{A}_{\mathbb{k}}^{2}\right)$ is described by the following classical theorem.

Theorem 1.4. (Jung [25], van der Kulk [49]) The automorphism group Aut $\left(\mathbb{A}_{\mathrm{k}}^{2}\right)$ is the free product of the subgroups $\mathrm{JONQ}^{+}\left(\mathbb{A}_{\mathbb{k}}^{2}\right)$ and $\mathrm{Aff}\left(\mathbb{A}_{\mathbb{k}}^{2}\right)$ amalgamated over their intersection $\operatorname{Aff}^{+}\left(\mathbb{A}_{\mathbb{k}}^{2}\right)$ :

$$
\operatorname{Aut}\left(\mathbb{A}_{\mathbb{k}}^{2}\right)=\operatorname{JONQ}^{+}\left(\mathbb{A}_{\mathbb{k}}^{2}\right) *_{\operatorname{Aff}^{+}\left(\mathbb{A}_{\mathrm{k}}^{2}\right)} \operatorname{Aff}\left(\mathbb{A}_{\mathbb{k}}^{2}\right)
$$

Remarks 1.5. 1. In fact Jung [25] just established, over a field $\mathbb{k}$ of characteristic 0, the equality

$$
\operatorname{Aut}\left(\mathbb{A}_{\mathbb{k}}^{2}\right)=\left\langle U^{+}, \operatorname{Aff}\left(\mathbb{A}_{\mathbb{k}}^{2}\right)\right\rangle
$$

where

$$
U^{+}=\left\{\Phi \in \mathrm{JONQ}^{+}\left(\mathbb{A}_{\mathrm{k}}^{2}\right) \mid \Phi:(x, y) \mapsto(x+f(y), y)\right\} .
$$

However, $\operatorname{Aut}\left(\mathbb{A}_{\mathbb{k}}^{2}\right) \neq U^{+} *_{U^{+} \cap \operatorname{Aff}\left(\mathbb{A}_{\mathrm{k}}^{2}\right)} \operatorname{Aff}\left(\mathbb{A}_{\mathbb{k}}^{2}\right)$, see Remark in $[27, \S 2]$.

Over an arbitrary ground field, van der Kulk did not formulate the theorem in terms of amalgamated free products, but from his results the theorem can be deduced readily, as this is done in [39] or [27, Theorem 2]. In characteristic zero, the theorem is a consequence of the Abhyankar-Moh-Suzuki theorem 1.1. See also [2], [10], [14], [15], [18], [21], [33], [35], [36], [41], [45], [48], and [52] for different approaches.

2. Actually we have

$$
\operatorname{Aut}\left(\mathbb{A}_{\mathbb{k}}^{2}\right)=\left\langle\operatorname{JONQ}^{+}\left(\mathbb{A}_{\mathbb{k}}^{2}\right), \tau\right\rangle
$$

where $\tau \in \operatorname{Aff}\left(\mathbb{A}_{\mathbb{k}}^{2}\right), \tau:(x, y) \longmapsto(y, x)$ is a twist. Notice that $\tau \in\left\langle\operatorname{Aff}^{+}\left(\mathbb{A}_{\mathbb{k}}^{2}\right), \operatorname{Aff}^{-}\left(\mathbb{A}_{\mathbb{k}}^{2}\right)\right\rangle$ and

$$
\operatorname{JONQ}^{-}\left(\mathbb{A}_{\mathbb{k}}^{2}\right)=\tau \operatorname{JONQ}^{+}\left(\mathbb{A}_{\mathbb{k}}^{2}\right) \tau, \quad \operatorname{Aff}^{-}\left(\mathbb{A}_{\mathbb{k}}^{2}\right)=\tau \operatorname{Aff}^{+}\left(\mathbb{A}_{\mathbb{k}}^{2}\right) \tau, \quad \text { and } \quad U^{-}=\tau U^{+} \tau,
$$

where the subgroup $U^{-} \subseteq \mathrm{JONQ}^{-}\left(\mathbb{A}_{\mathbb{k}}^{2}\right)$ is defined similarly as $U^{+}$. In particular

$$
\operatorname{Aut}\left(\mathbb{A}_{\mathbb{k}}^{2}\right)=\left\langle\operatorname{JONQ}^{+}\left(\mathbb{A}_{\mathbb{k}}^{2}\right), \operatorname{JONQ}^{-}\left(\mathbb{A}_{\mathbb{k}}^{2}\right)\right\rangle \text {. }
$$

The following theorem absorbed several previously known results. In this generality, it was first proved by Kambayashi [28] (using a result of Wright [50]) as a consequence of the Jung-van der Kulk theorem 1.4.

Theorem 1.6. (Kambayashi [28, Theorem 4.3], Wright [50], [51]) Any algebraic subgroup of the group $\operatorname{Aut}\left(\mathbb{A}_{\mathbb{k}}^{2}\right)$ is conjugate either to a subgroup of $\operatorname{Aff}\left(\mathbb{A}_{\mathbb{k}}^{2}\right)$, or to a subgroup of $\mathrm{JONQ}^{+}\left(\mathbb{A}_{\mathbb{k}}^{2}\right)$.

The proof exploites the following observation: every algebraic subgroup of $\operatorname{Aut}\left(\mathbb{A}_{\mathbb{k}}^{2}\right)$ has bounded degree, hence also a bounded length with respect to the free amalgamated product structure. However, by Serre [46] a subgroup of bounded length in an amalgamated free product $A *_{C} B$ is conjugate to a subgroup of one of the factors $A$ and $B$.

In the next corollary we suppose that the base field $\mathbb{k}$ is algebraically closed of characteristic 0 (while certain assertions remain valid in the positive characteristic case). 
Corollary 1.7. 1. (Igarashi [24], Furushima [17]) Every finite subgroup of Aut $\left(\mathbb{A}_{\mathrm{k}}^{2}\right)$ is conjugate to a subgroup of $\mathrm{GL}(2, \mathbb{k})$.

2. (Gutwirth [22], Bialynicki-Birula [9]) Every maximal torus in Aut $\left(\mathbb{A}_{\mathrm{k}}^{2}\right)$ has rank 2 and is conjugate to the standard maximal torus $\mathbb{T} \subseteq \mathrm{GL}(2, \mathbb{k})$.

3. (Gutwirth [22], Bialynicki-Birula [9]) Every one-torus in $\operatorname{Aut}\left(\mathbb{A}_{\mathbb{k}}^{2}\right)$ is conjugate to a subtorus of $\mathbb{T}$.

4. (Rentschler [45]) Every $\mathbb{G}_{a}(\mathbb{k})$-action on $\mathbb{A}_{\mathbb{k}}^{2}$ is conjugate to an action via de Jonqières transformations

$$
t .(x, y)=(x+t f(y), y), \quad \text { where } t \in \mathbb{G}_{a}(\mathbb{k}) \text { and } f \in \mathbb{k}[y] .
$$

Remarks 1.8. 1. Assertions (1)-(3) follow from a more general result for reductive groups, see 2.5 below.

2. Analogs of (1) and (4) fail in higher dimensions, while (3) holds in dimension 3 and is open in higher dimensions. We do not dwell on this here (see, however, [26], [43] and the survey [30]; see also [7] and [38] for the case of a positive characteristic).

\section{Subgroups of DE Jonquères Group AND StABILIZERs of Plane CuRVES}

2.1. Subgroups of the de Jonquères group. In this subsection the base field $\mathbb{k}$ will be an algebraically closed field of characteristic zero (while some results are still valid over an arbitrary field of characteristic zero.) By abuse of language, we still call de Jonquères groups the subgroups $\operatorname{Jonq}^{ \pm}\left(\mathbb{A}_{\mathbb{k}}^{2}\right) \subseteq \operatorname{JONQ}^{ \pm}\left(\mathbb{A}_{\mathbb{k}}^{2}\right)$ consisting, respectively, of the transformations

$$
\varphi^{+}:(x, y) \longmapsto(\alpha x+f(y), \beta y),
$$

and

$$
\varphi^{-}:(x, y) \longmapsto(\alpha x, \beta y+f(x)),
$$

where $\alpha, \beta \in \mathbb{k}^{\times}$and $f \in \mathbb{k}[t]$ (so $\Phi^{ \pm}$as in (3), (4) belongs to $\operatorname{Jonq}^{ \pm}\left(\mathbb{A}_{\mathbb{k}}^{2}\right)$ if and only if $q=0$ ). Clearly,

$$
\operatorname{Jonq}^{+}\left(\mathbb{A}_{\mathbb{k}}^{2}\right) \simeq U^{+} \rtimes \mathbb{T},
$$

where $\mathbb{T} \subseteq \mathrm{GL}(2, \mathbb{k})$ is the maximal torus

$$
\mathbb{T}=\left\{\delta \in \mathrm{Jonq}^{+}\left(\mathbb{A}_{\mathbb{k}}^{2}\right) \mid \delta:(x, y) \mapsto(\alpha x, \beta y)\right\},
$$

and $U^{+} \simeq \mathbb{k}[t]$ as in (6) is an infinite dimensional vector group. Indeed, let $\rho: \operatorname{Jonq}^{+}\left(\mathbb{A}_{\mathbb{k}}^{2}\right) \rightarrow \mathbb{T}$ denote the canonical surjection provided by (9). Then any element $\varphi^{+} \in \operatorname{Jonq}^{+}\left(\mathbb{A}_{\mathrm{k}}^{2}\right)$ as in (7) admits a decomposition $\varphi^{+}=\partial \circ \delta$, where $\delta=\rho\left(\varphi^{+}\right) \in \mathbb{T}$ and $\partial \in U^{+}, \partial:(x, y) \mapsto$ $(x+f(y / \beta), y)$.

In particular, $\mathrm{Jonq}^{+}\left(\mathbb{A}_{\mathrm{k}}^{2}\right)$ is a metabelian group, and $U^{+}$can be considered as its unipotent radical in the following sense. There is a natural filtration by algebraic subgroups

$$
\operatorname{Jonq}^{+}\left(\mathbb{A}_{\mathbb{k}}^{2}\right)=\bigcup_{n \in \mathbb{N}} \operatorname{Jonq}_{n}^{+}\left(\mathbb{A}_{\mathbb{k}}^{2}\right)
$$

where

$$
\operatorname{Jonq}_{n}^{+}\left(\mathbb{A}_{\mathrm{k}}^{2}\right) \simeq U_{n}^{+} \rtimes \mathbb{T}
$$

consists of all elements $\varphi^{+} \in \mathrm{Jonq}^{+}\left(\mathbb{A}_{\mathbb{k}}^{2}\right)$ with $\operatorname{deg} f \leq n$. Then $\mathbb{T}$ is a maximal torus of $\mathrm{Jonq}_{n}^{+}\left(\mathbb{A}_{\mathbb{k}}^{2}\right)$, and $U_{n}^{+}=U^{+} \cap \mathrm{Jonq}_{n}^{+}\left(\mathbb{A}_{\mathbb{k}}^{2}\right)$ is its unipotent radical. 
Any algebraic subgroup $G$ of $\mathrm{Jonq}^{+}\left(\mathbb{A}_{\mathbb{k}}^{2}\right)$ has finite degree

$$
d=\min \left\{n \mid G \subseteq \operatorname{Jonq}_{n}^{+}\left(\mathbb{A}_{\mathbb{k}}^{2}\right)\right\} .
$$

In particular, every maximal torus in $\mathrm{Jonq}^{+}\left(\mathbb{A}_{\mathbb{k}}^{2}\right)$ is conjugate with $\mathbb{T}$, and any algebraic subgroup which consists of semi-simple elements is conjugate to a subgroup of $\mathbb{T}$ (see [23, VII.19.4, VIII.21.3A]).

In the following lemma we characterize semi-simple and torsion elements in the group $\operatorname{Jonq}^{+}\left(\mathbb{A}_{\mathbb{k}}^{2}\right)$.

Lemma 2.1. (a) An element $\varphi^{+} \in \mathrm{Jonq}^{+}\left(\mathbb{A}_{\mathbb{k}}^{2}\right)$ as in (7) is semi-simple if and only if the polynomial $f(y)=\sum_{m \geq 0} a_{m} y^{m}$ satisfies the condition

$$
a_{m}=0 \quad \text { if } \quad \alpha=\beta^{m},
$$

if and only if there exists $\mu \in U^{+}$such that $\mu^{-1} \varphi^{+} \mu=\delta \in \mathbb{T}^{3}$.

(b) An element $\varphi^{+} \in \operatorname{Jonq}^{+}\left(\mathbb{A}_{\mathbb{k}}^{2}\right)$ as in (7) is of finite order if and only if it is semi-simple and $\delta=\rho\left(\varphi^{+}\right) \in \mathbb{T}$ is of finite order.

Proof. (a) We claim that if $\varphi^{+}$satisfies (10) then there exists $\mu \in U^{+}, \mu:(x, y) \mapsto(x+g(y), y)$, where $g \in \mathbb{k}[t]$, such that

$$
\mu^{-1} \varphi^{+} \mu=\delta=\rho\left(\varphi^{+}\right) \in \mathbb{T} \quad \text { or, equivalently, } \partial=[\mu, \delta]=\mu \delta \mu^{-1} \delta^{-1} .
$$

Writing $g(y)=\sum_{m \geq 0} b_{m} y^{m}$, it is readily seen that $\mu$ satisfies (11) if and only if the coefficients $b_{m}$ of $g$ satisfy the conditions

$$
b_{m}= \begin{cases}\frac{a_{m}}{\beta^{m}-\alpha} & \text { if } \beta^{m}-\alpha \neq 0 \\ \text { arbitrary } & \text { if } \beta^{m}-\alpha=0=a_{m} .\end{cases}
$$

Indeed, (11) can be written as

$$
(x+g(y)-\alpha g(y / \beta), y)=(x+f(y / \beta), y)
$$

which is equivalent to (12). This shows the existence of $\mu$ in (11) under condition (10).

On the other hand, if (10) fails i.e., there exists $m \in \mathbb{N}$ such that $\beta^{m}-\alpha=0$ and $a_{m} \neq 0$, then $\mu$ as in (11) cannot exist. The remaining claims in (a) are easy and so we leave them to the reader.

(b) We have to show that if $\left(\varphi^{+}\right)^{k}=$ id then (10) holds and $\delta^{k}=\mathrm{id}$, and vice versa. Indeed, letting $\gamma=\varphi^{+}$for any $k \geq 1$ we obtain

$$
\gamma^{k}:(x, y) \longmapsto\left(\alpha^{k} x+f_{k}(y), \beta^{k} y\right)
$$

where

$$
f_{k}(y)=\alpha^{k-1} f(y)+\alpha^{k-2} f(\beta y)+\ldots+f\left(\beta^{k-1} y\right) .
$$

Therefore $\gamma^{k}=$ id if and only if $\alpha^{k}=\beta^{k}=1$ (i.e. $\delta^{k}=$ id) and $f_{k}=0$. However, $f_{k}=0$ if and only if

$$
\forall m \geq 0, \quad \text { either } \quad a_{m}=0 \quad \text { or } \quad \alpha^{k-1}+\alpha^{k-2} \beta^{m}+\ldots+\beta^{m(k-1)}=0 .
$$

The latter equality can be written as

$$
\left(\alpha / \beta^{m}\right)^{k-1}+\left(\alpha / \beta^{m}\right)^{k-2}+\ldots+1=0 .
$$

\footnotetext{
${ }^{3}$ In fact here $\delta=\rho\left(\varphi^{+}\right)$.
} 
Since $\left(\alpha / \beta^{m}\right)^{k}=1$ this holds if and only if $\alpha \neq \beta^{m}$.

Lemma 2.1(b) admits the following interpretation.

Remark 2.2. Consider a $\mathbb{Z} / d \mathbb{Z}$-grading on the polynomial ring $A=\mathbb{k}[t]$ :

$$
A=\bigoplus_{i=0}^{d-1} A_{d, i}, \quad \text { where } \quad A_{d, i}=t^{i} \mathbb{k}\left[t^{d}\right] .
$$

According to this decomposition any element $f \in A$ can be written as $f=\sum_{i=0}^{d-1} f_{i}$, where $f_{i} \in A_{i} \forall i$. Assuming that $\beta$ has finite order $d$ we let $m(\alpha, \beta)=\min \left\{m \geq 0 \mid \alpha=\beta^{m}\right\}$. Then condition (10) can be expressed as $f_{m(\alpha, \beta)}=0$, provided this quantity is well defined. (Otherwise (10) does not impose any condition.) This phenomenon can be seen on the following simple examples.

Example 2.3. Letting $d=2$ any element $f \in \mathbb{k}[t]$ can be written as $f=f_{0}+f_{1}$, where $f_{0}$ is even and $f_{1}$ is odd. There are the following three types of involutions $\varphi^{+}:(x, y) \mapsto$ $(\alpha x+f(y), \beta y)$ in $\operatorname{Jonq}^{+}\left(\mathbb{A}_{\mathrm{k}}^{2}\right)$ :

1. $\alpha=1, \beta=-1, f \in \mathbb{k}[t]$ is odd i.e. $f_{0}=0$;

2. $\alpha=-1, \beta=1, f \in \mathbb{k}[t]$ is arbitrary;

3. $\alpha=-1, \beta=-1, f \in \mathbb{k}[t]$ is even i.e. $f_{1}=0$.

Lemma 2.4. Consider a pair of elements $\gamma, \tilde{\gamma} \in \operatorname{Jonq}^{+}\left(\mathbb{A}_{\mathbb{k}}^{2}\right)$,

$$
\gamma:(x, y) \mapsto(\alpha x+f(y), \beta y) \quad \text { and } \quad \tilde{\gamma}:(x, y) \mapsto(\tilde{\alpha} x+\tilde{f}(y), \tilde{\beta} y) \text {, }
$$

where

$$
f(y)=\sum_{m \geq 0} a_{m} y^{m} \quad \text { and } \quad \tilde{f}(y)=\sum_{m \geq 0} \tilde{a}_{m} y^{m} .
$$

Then $\gamma$ and $\tilde{\gamma}$ commute if and only if

$$
a_{m}\left(\tilde{\beta}^{m}-\tilde{\alpha}\right)=\tilde{a}_{m}\left(\beta^{m}-\alpha\right) \quad \forall m \geq 0 .
$$

Proof. The proof is easy and is left to the reader.

Recall that a quasitorus is a product of a torus and a finite abelian group. Any algebraic subgroup of a torus is a quasitorus.

Proposition 2.5. Any reductive algebraic subgroup $G$ of the group $\mathrm{Jonq}^{+}\left(\mathbb{A}_{\mathbb{k}}^{2}\right)$ is conjugate to a subgroup of the torus $\mathbb{T}$. More precisely, there exists an element $\mu \in U^{+}$such that

$$
\mu^{-1} \circ \varphi \circ \mu=\rho(\varphi) \quad \forall \varphi \in G,
$$

where

$$
\rho: \operatorname{Jonq}^{+}\left(\mathbb{A}_{\mathbb{k}}^{2}\right) \rightarrow \mathbb{T}=\operatorname{Jonq}^{+}\left(\mathbb{A}_{\mathbb{k}}^{2}\right) / U^{+}
$$

is the natural surjection. In particular $\mu^{-1} G \mu=\rho(G) \subseteq \mathbb{T}$.

Proof. Since $G$ is reductive the unipotent radical $R_{u}(G)$ is trivial. Hence also the subgroup $G \cap U^{+}$is trivial. Thus $\rho$ restricts to an isomorphism $\rho \mid G: G \stackrel{\simeq}{\longrightarrow} \rho(G) \subseteq \mathbb{T}$. In particular, $G$ is abelian and consists of semi-simple elements, cf. lemma 2.1. By [23, VII.19.4, VIII.21.3A] $G$ is contained in a maximal torus $\mathbb{T}^{\prime}$ conjugate to $\mathbb{T}$. Now the first assertion follows. 
Let us show the second. Since $G$ is abelian and consists of semi-simple elements, (10) and (13) are fulfilled for any pair of elements $\gamma, \tilde{\gamma} \in G$. Thus there is $\mu \in U^{+}$satisfying (12) and then also (11) for all $\varphi \in G$ simultaneously (see the proof of lemma 2.1). This $\mu$ is as desired.

As an application we can deduce the following well known fact (see [31, Theorem 2]).

Corollary 2.6. Every effective action of a reductive algebraic group $G$ on the affine plane $\mathbb{A}_{\mathbb{k}}^{2}$ is linearizable. In other words, $G$ is conjugate in the group $\operatorname{Aut}\left(\mathbb{A}^{2}\right)$ to a subgroup of $\mathrm{GL}(2, \mathbb{k})$.

Let us provide an argument following an indication in [31].

Proof. By theorem 1.6 $G$ is conjugate in $\operatorname{Aut}\left(\mathbb{A}^{2}\right)$ to a subgroup of one of the groups $\operatorname{Aff}\left(\mathbb{A}_{\mathrm{k}}^{2}\right)$ and $\mathrm{Jonq}^{+}\left(\mathbb{A}_{\mathrm{k}}^{2}\right)$. In the latter case by proposition $2.5 G$ is conjugate to a subgroup of the torus $\mathbb{T}$ contained in $\operatorname{Aff}\left(\mathbb{A}_{\mathbb{k}}^{2}\right)$. Thus we may assume that $G \subseteq \operatorname{Aff}\left(\mathbb{A}_{\mathbb{k}}^{2}\right)$.

It remains to show that $G$ has a fixed point in $\mathbb{A}^{2}$. Observe that $G \subseteq \operatorname{Aff}\left(\mathbb{A}_{\mathbb{k}}^{2}\right)$ admits a representation in $\operatorname{GL}(3, \mathbb{k})$ by matrices of the form $\left(\begin{array}{ccc}* & * & * \\ * & * & * \\ 0 & 0 & 1\end{array}\right)$. Since $G$ is reductive and char $(\mathbb{k})=0, G$ is geometrically reductive. Therefore the $G$-invariant plane $L_{0}=\left\{x_{3}=0\right\}$ in $\mathbb{A}_{\mathbb{k}}^{3}$ with coordinates $\left(x_{1}, x_{2}, x_{3}\right)$ has a $G$-invariant complement, say, $R$, which meets the parallel $G$-invariant plane $L_{1}=\left\{x_{3}=1\right\}$ in a fixed vector. This yields a fixed point of $G$ in $\mathbb{A}_{\mathrm{k}}^{2} \simeq_{G} L_{1}$. Now the proof is completed.

Remark 2.7. Due to corollary 2.6, when dealing with quotients of the affine plane by finite group actions it suffices to restrict to linear such actions.

2.2. Stabilizers of acyclic plane curves. In the sequel $\mathbb{A}^{n}$ stands for the affine $n$-space over $\mathbb{C}$. For an algebraic curve $C$ in $\mathbb{A}^{2}$ we let $\operatorname{Stab}(C)$ be the stabilizing group, or stabilizer of $C$ i.e. the group of all algebraic automorphisms of $\mathbb{A}^{2}$ that preserve $C$ :

$$
\operatorname{Stab}(C)=\left\{\gamma \in \operatorname{Aut}\left(\mathbb{A}^{2}\right) \mid \gamma(C)=C\right\}
$$

In this subsection we describe the stabilizers of acyclic plane curves given in one of the canonical forms (1) and (2) of theorem 1.3(b). Accordingly, we distinguish the following six types of acyclic curves $C$ :

(I) $C$ is irreducible and smooth, and then $C \sim C_{y}$;

(II) $C$ consists of two smooth, mutually transversal components, and then $C \sim C_{x} \cup C_{y}$;

(III) $C$ has $r \geq 2$ singular points and so is equivalent to a union of $r$ parallel lines and a transversal line given by (1) with $\varepsilon_{y}=1$;

(IV) $C$ has an ordinary singularity of multiplicity $r \geq 3$ and so is equivalent to a union of $r$ distinct lines through the origin;

(V) All irreducible components of $C$ are smooth and $C$ has a non-ordinary singular point. So $C$ is equivalent to a curve given by equation (2) with $a=1<b$ and $\varepsilon_{y}+r \geq 2$;

(VI) $C$ contains a singular component, and then it is equivalent to a curve given by (2) with $\min \{a, b\}>1$ and $r \geq 1$.

We analyse each of these cases separately. 
In the next proposition we study the curves of type (I). We show that the stabilizer $\operatorname{Stab}\left(C_{y}\right)$ of the coordinate axis $C_{y}=\{y=0\}$ in $\mathbb{A}^{2}$ consists of the de Jonquières transformations. For the acyclic curves of types (II)-(IV) reduced to the canonical form the stabilizer is described in corollaries 2.9 and 2.10, and for those of types (V) and (VI) in propositions 2.13 and 2.14.

Proposition 2.8. The stabilizer $\operatorname{Stab}\left(C_{y}\right)$ in $\operatorname{Aut}\left(\mathbb{A}^{2}\right)$ coincides with the subgroup $\mathrm{Jonq}^{+}\left(\mathbb{A}^{2}\right)$, while $\operatorname{Stab}\left(C_{x}\right)=\operatorname{Jonq}^{-}\left(\mathbb{A}^{2}\right)$.

Proof. Every $\gamma \in \operatorname{Stab}\left(C_{y}\right)$ sends $y$ to $\beta y$ for some $\beta \in \mathbb{C}^{\times}$. Up to an affine transformation we may assume that $\beta=1$ and $\left.\gamma\right|_{C_{y}}=\operatorname{id}_{C_{y}}$. Suppose that $\gamma$ sends $x$ to $x+h(x, y)$. Since $h(x, 0)=0$ we have $h(x, y)=y p(x, y)$. To show that $p(x, y)$ does not depend on $x$ we write

$$
p(x, y)=a_{0}(y)+a_{1}(y) x+\ldots+a_{k}(y) x^{k} \quad \text { with } \quad a_{k}(y) \neq 0 .
$$

Clearly, $\gamma$ preserves every line $y=y_{0}$ and induces an affine automorphism of this line. Picking $y_{0}$ with $a_{k}\left(y_{0}\right) \neq 0$ we get $k \leq 1$. Letting $k=1$ we obtain $\gamma: x \mapsto\left(1+y a_{1}(y)\right) x+y a_{0}(y)$. If $y_{1}$ is a root of the non-constant polynomial $1+y a_{1}(y)$ then $\gamma$ induces a constant map on the line $y=y_{1}$, a contradiction. Hence $k=0$. Thus $\operatorname{Stab}\left(C_{y}\right)=\operatorname{Jonq}^{+}\left(\mathbb{A}^{2}\right)$. The proof of the second assertion is similar.

In the following two corollaries we describe the stabilizers of the canonical curves of types (II)-(IV).

Corollary 2.9. If $C=\bigcup_{i=1}^{r} L^{i}$ is a union of $r \geq 2$ affine lines in $\mathbb{A}^{2}$ through the origin then $\operatorname{Stab}(C) \subseteq \mathrm{GL}(2, \mathbb{C})$.

Proof. We may suppose that $L_{1}=C_{x}$ and $L_{2}=C_{y}$. For any $g \in \operatorname{Stab}(C)$ we can find $h \in \mathrm{GL}(2, \mathbb{C})$ such that $g\left(L_{i}\right)=h\left(L_{i}\right), i=1,2$. It follows by proposition 2.8 that $\gamma=h^{-1} g \in$ $\mathrm{GL}(2, \mathbb{C})$. Hence also $g=h \gamma \in \mathrm{GL}(2, \mathbb{C})$.

The following corollary is immediate.

Corollary 2.10. (a) Let $C=\{f(y)=0\}$, where $f \in \mathbb{C}[y]$ is a polynomial of degree $\geq 2$ with simple roots. If $K \subseteq \mathbb{C}$ denotes the set of these roots, then

$$
\operatorname{Stab}(C)=\mathbb{T}_{1,0} \cdot U^{+} \cdot \operatorname{Stab}(K),
$$

where $U^{+} \subseteq \operatorname{Aut}\left(\mathbb{A}^{2}\right)$ is as in (6),

$$
\mathbb{T}_{1,0}=\left\{\lambda \in \mathbb{T} \mid \lambda:(x, y) \mapsto(\alpha x, y), \alpha \in \mathbb{C}^{\times}\right\},
$$

and the stabilizer $\operatorname{Stab}(K) \subseteq \operatorname{Aut}\left(\mathbb{A}^{1}\right) \hookrightarrow \operatorname{Aut}\left(\mathbb{A}^{2}\right)$ acts naturally on the symbol $y$.

(b) If $C$ of type (II) is the coordinate cross $\{x y=0\}$ in $\mathbb{A}^{2}$ then $\operatorname{Stab}(C)=N(\mathbb{T})$ is the normalizer of the maximal torus $\mathbb{T}$ in the group $\mathrm{GL}(2, \mathbb{C})$.

(c) If $C$ of type (IV) is a union of $r$ affine lines through the origin, where $r \geq 3$, then $\operatorname{Stab}(C) \subseteq \mathrm{GL}(2, \mathbb{C})$ is a finite extension of the group $\mathbb{T}_{1,1}=\mathbb{C}^{\times} \cdot \mathrm{id}$ of scalar matrices.

(d) If $C$ of type (III) is given by equation $x y f(x)=0$, where $f \in \mathbb{C}[x]$ is a polynomial of degree $\geq 1$ with simple roots such that $f(0) \neq 0$, then $\operatorname{Stab}(C) \subseteq \mathbb{T}$ is a finite extension of the one-torus $\mathbb{T}_{0,1} \subseteq \mathbb{T}$.

Thus in (b)-(d) the stabilizer $\operatorname{Stab}(C)$ is a linear group, while the group in (a) is infinite dimensional. The group $\operatorname{Stab}(C)$ in (b) is nonabelian. It can occur to be nonabelian also in (c), as in the following simple example. 
Example 2.11. Given a nonabelian finite subgroup $G \subseteq \mathrm{GL}(2, \mathbb{C})$ consider the curve $C=$ $\bigcup_{g \in G} g\left(C_{y}\right)$. Since $G \subseteq \operatorname{Stab}(C)$ the latter group is nonabelian.

Consider further an irreducible acyclic curve $C_{a, b}$ given in $\mathbb{A}^{2}$ by equation $y^{a}-x^{b}=0$, where $a, b \geq 1$ and $\operatorname{gcd}(a, b)=1$. In the following proposition we describe the stabilizer $\operatorname{Stab}\left(C_{a, b}\right)$ for a singular such curve. Consider a one-parameter subgroup $\mathbb{T}_{a, b}$ of the torus $\mathbb{T}$,

$$
\mathbb{T}_{a, b}=\left\{\gamma_{a, b}(t) \mid t \in \mathbb{G}_{m}\right\} \subseteq \operatorname{Stab}\left(C_{a, b}\right), \quad \text { where } \quad \gamma_{a, b}(t):(x, y) \longmapsto\left(t^{a} x, t^{b} y\right) .
$$

Proposition 2.12. If $\min \{a, b\}>1$ then $\operatorname{Stab}\left(C_{a, b}\right)=\mathbb{T}_{a, b}$.

Proof. Letting $C=C_{a, b}$ and $\Gamma=\operatorname{Stab}(C)$, we consider the pointwise stabilizer

$$
\Gamma_{0}=\left\{\gamma \in \Gamma|\gamma|_{C}=\operatorname{id}_{C}\right\} \subseteq \Gamma .
$$

Claim 1. The group $\Gamma_{0}$ is torsion free.

Proof of claim 1. Let $\gamma_{0} \neq$ id be an element of finite order in $\Gamma_{0}$. The finite cyclic group $\left\langle\gamma_{0}\right\rangle$ is reductive. Hence the fixed point locus $\left(\mathbb{A}^{2}\right)^{\gamma_{0}}$ is smooth by the Luna Étale Slice Theorem [34] (see also [44, §6]). Thus an irreducible component $C=C_{a, b}$ of this locus must be smooth as well. However, under our assumptions the curve $C_{a, b}$ is singular.

An element $\gamma \in \Gamma$ sends the polynomial $q(x, y)=y^{a}-x^{b}$ to $\lambda_{\gamma} q$, where $\lambda_{\gamma} \in \mathbb{G}_{m}$. Letting $\psi(\gamma)=\lambda_{\gamma}$ yields a character $\psi: \Gamma \rightarrow \mathbb{G}_{m}$ of $\Gamma$. The following claim is immediate.

Claim 2. $\Gamma=\mathbb{T}_{a, b} \cdot \Gamma_{1}$, where $\Gamma_{1}=\operatorname{ker}(\psi)$.

Claim 3. The kernel $\Gamma_{1}=\operatorname{ker}(\psi)$ is a torsion group. Furthermore, there is a positive integer $N$ such that the orders of all elements in $\Gamma_{1}$ divide $N$.

Proof of claim 3. The group $\Gamma_{1}$ acts on every fiber $C_{\alpha}=\{q(x, y)=\alpha\}$ of $q$. Since for $\alpha \neq 0$ the affine curve $C_{\alpha}$ has positive genus, its automorphism group is finite of order, say, $N$. For any $\alpha \neq 0 \neq \beta$ the curves $C_{\alpha}$ and $C_{\beta}$ are isomorphic. So their automorphism groups are isomorphic, too. Since $\left.\gamma^{N}\right|_{C_{\alpha}}=\operatorname{id}_{C_{\alpha}}$ for every $\gamma \in \Gamma_{1}$ and $\alpha \neq 0$, we have $\gamma^{N}=$ id.

Claim 4. $\Gamma_{0}=\{$ id $\}$.

Proof of claim 4. According to claim 3 we have $\Gamma_{0} \subseteq \mathbb{T}_{a, b} \cdot \Gamma_{1}$. Writing an element $\gamma_{0} \in \Gamma_{0}$ as $\gamma_{0}=\gamma_{a, b}(t) \circ \gamma_{1}$, from $\left.\gamma_{0}\right|_{C}=\operatorname{id}_{C}$ we obtain $\left.\gamma_{1}^{-1}\right|_{C}=\left.\gamma_{a, b}(t)\right|_{C}$. Hence $\operatorname{id}_{C}=\left.\gamma_{1}^{-N}\right|_{C}=$ $\left.\gamma_{a, b}\left(t^{N}\right)\right|_{C}$. It follows that $t^{N}=1$. Since $\Gamma_{0} \cap \Gamma_{1}=\{$ id $\}$ the map $\left.\psi\right|_{\Gamma_{0}}: \Gamma_{0} \rightarrow \mathbb{G}_{m}$ is injective. So $\psi\left(\gamma_{0}\right)=\psi\left(\gamma_{a, b}(t)\right)$ has finite order dividing $N$. Due to claim 1 we can conclude that $\Gamma_{0}=\{\mathrm{id}\}$.

Claim 5. $\Gamma=\mathbb{T}_{a, b}$.

Proof of claim 5. For any $\gamma \in \Gamma$ there exists $t \in \mathbb{C}^{\times}$such that $\gamma\left|C=\gamma_{a, b}(t)\right| C$. Hence $\gamma \circ \gamma_{a, b}^{-1}(t) \in \Gamma_{0}=\{\mathrm{id}\}$ and so $\gamma=\gamma_{a, b}(t) \in \mathbb{T}_{a, b}$.

This ends the proof.

Using propositions 2.8 and 2.12 we describe in 2.13 and 2.14 below the structure of the stabilizer $\operatorname{Stab}(C)$ for reduced (but possibly reducible) acyclic plane curves $C$ of the remaining types (VI) and (V), respectively.

Proposition 2.13. Let $C$ be an acyclic curve of type (VI) given by equation (2), where $r \geq 1$ and $\operatorname{gcd}(a, b)=1$. If $\min \{a, b\}>1$ then $\operatorname{Stab}(C)$ is a quasitorus of rank 1 contained in the maximal torus $\mathbb{T}$. 
Proof. Let $C^{i}=\left\{y^{a}-\kappa_{i} x^{b}=0\right\}, i=1, \ldots, r$, be the irreducible components of the curve $C$. Clearly $\mathbb{T}_{a, b} \subseteq \operatorname{Stab}(C)$. If $r=1$ then by proposition $2.12 \operatorname{Stab}(C)=\mathbb{T}_{a, b}$. Suppose that $r>1$. Consider a finite abelian group $H=\operatorname{Stab}(C) \cap \mathbb{T}_{1,0}$. We claim that $\operatorname{Stab}(C)=H \cdot \mathbb{T}_{a, b} \subseteq \mathbb{T}$. Hence this is a quasitorus of rank one, as stated. Indeed, if $\delta \in \operatorname{Stab}(C) \backslash \mathbb{T}_{a, b}$ then $\delta\left(C^{1}\right)=C^{i}$ for some $i>1$. If $h \in \mathbb{T}_{1,0}$ is such that $h\left(C^{i}\right)=C^{1}$ then $\gamma=h \circ \delta \in \operatorname{Stab}\left(C^{1}\right)=\mathbb{T}_{a, b}$. Hence $h=\gamma \circ \delta^{-1} \in H$ and so $\delta=h^{-1} \circ \gamma \in H \cdot \mathbb{T}_{a, b}$. Now the claim follows. This ends the proof.

Proposition 2.14. Let $C$ be an acyclic curve $C$ of type ( $V$ ) given by equation (2), where $r \geq 1$ and $\varepsilon_{y}+r \geq 2$. If $a=1<b$ then $\operatorname{Stab}(C)$ is a quasitorus of rank 1 conjugated in the group Aut $\left(\mathbb{A}^{2}\right)$ to a subgroup of the torus $\mathbb{T}$.

Proof. If $\varepsilon_{x}=1$ the proof is easy and can be left to the reader (cf. corollary 2.10(b)). Thus we may restrict to the case $\varepsilon_{x}=0$. By our assumptions $C$ is reducible and all components $C^{i}$ of $C$ are smooth and mutually tangent at the origin (we let here $C^{0}=C_{y}$ if $\varepsilon_{y}=1$ ). Consider the pencil $\mathcal{L}=\left\{C_{\mu}\right\}_{\mu \in \mathbb{P}^{1}}$, where $C_{\mu}=\left\{y-\mu x^{b}=0\right\}$ for $\mu \neq \infty$ and $C_{\infty}=C_{x}$.

Claim 1. The pencil $\mathcal{L} \backslash\left\{C_{x}\right\}$ is stable under the action of the group $\operatorname{Stab}(C)$ on $\mathbb{A}^{2}$.

Proof of claim 1. The unique singular point $\overline{0} \in C$ is fixed under the action of $\operatorname{Stab}(C)$. Furthermore, for every $g \in \operatorname{Stab}(C)$ and every $\mu \in \mathbb{C}$, either $C_{\mu} \subseteq C$ or $g\left(C_{\mu}\right) \cap C=\{\overline{0}\}$. In the latter case, letting $B_{\mu}=g\left(C_{\mu}\right)$ it follows that the restriction $\left.\left(y-\kappa_{i} x^{b}\right)\right|_{B_{\mu}}$ vanishes just at the origin. Hence in an affine coordinate, say, $z$ in $B_{\mu} \simeq \mathbb{A}^{1}$ centered at the origin the latter function is a monomial $\lambda_{i} z^{\alpha_{i}}$, where $\lambda_{i} \in \mathbb{C}^{\times}$and $\alpha_{i} \geq 1$. Therefore $B_{\mu}$ is a component of the curve

$$
\left(y-\kappa_{i} x^{b}\right)^{\alpha_{j}}-\delta\left(y-\kappa_{j} x^{b}\right)^{\alpha_{i}}=0, \quad \text { where } \quad \lambda_{i}^{\alpha_{j}}=\delta \lambda_{j}^{\alpha_{i}} .
$$

Since $B_{\mu}$ is smooth one of the exponents $\alpha_{i}, \alpha_{j}$ divides the other. Preservation of the local intersection indices under $g$ implies that $i\left(B_{\mu}, C^{i}, \overline{0}\right)=b \forall i$. It follows that actually $\alpha_{i}=\alpha_{j}$ $\forall i, j$. Finally $B_{\mu}$ coincides with a certain member $C_{\mu^{\prime}} \in \mathcal{L}$, as claimed.

Clearly $\nu(g): \mathbb{A}^{1} \rightarrow \mathbb{A}^{1}, \mu \mapsto \mu^{\prime}$, is an affine transformation leaving invariant the set $K=\left\{\kappa_{i}\right\} \subseteq \mathbb{A}^{1}$ enriched by $\kappa_{0}=0$ in case where $\varepsilon_{y}=1$ in $(7)$. Let $\operatorname{Stab}(K)$ be the stabilizer of $K$ in $\operatorname{Aut}\left(\mathbb{A}^{1}\right)$. The group $\operatorname{Stab}(K)$ fixes the isobarycentre of $K$ and so embeds in $\mathbb{G}_{m}$ onto a finite subgroup. The natural homomorphism $\varphi: \operatorname{Stab}(C) \rightarrow \operatorname{Stab}(K)$ fits in the exact sequence

$$
1 \rightarrow \operatorname{Stab}_{0}(C) \rightarrow \operatorname{Stab}(C) \stackrel{\varphi}{\longmapsto} \operatorname{Stab}(K) \rightarrow \ldots
$$

where $\operatorname{Stab}_{0}(C)=\operatorname{ker}(\varphi) \subseteq \operatorname{Stab}(C)$ consists of the elements $g \in \operatorname{Aut}\left(\mathbb{A}^{2}\right)$ leaving invariant every component $C^{i}$ of $C$.

Claim 2. $\operatorname{Stab}_{0}(C)=\mathbb{T}_{1, b}$.

Proof of claim 2. If $g \in \operatorname{Stab}_{0}(C)$ then $\varphi(g)=\mathrm{id}{ }_{K}$. Since $|K| \geq 2$ it follows that $\nu(g)=\mathrm{id}_{\mathbb{A}^{1}}$ i.e., $g\left(C_{\mu}\right)=C_{\mu} \forall \mu \in \mathbb{A}^{1}$. In particular, $g\left(C_{0}\right)=C_{0}$, where $C_{0}=C_{y}$. By proposition 2.8 we have $g:(x, y) \mapsto(\alpha x+f(y), \beta y)$ for some $\alpha, \beta \in \mathbb{C}^{\times}$and $f \in \mathbb{C}[y]$. The equality $g\left(C^{1}\right)=C^{1}$ implies that $f=0$ and $\beta=\alpha^{b}$, that is, $g \in \mathbb{T}_{1, b}$. Now the claim follows.

Thus $\operatorname{Stab}(C)$ is an extension of the one-torus $\mathbb{T}_{1, b}$ by a finite cyclic group. The proof can be completed due to the following

Claim 3. $\operatorname{Stab}(C)$ is conjugated in $\operatorname{Aut}\left(\mathbb{A}^{2}\right)$ to a subgroup of the maximal torus $\mathbb{T}$.

Proof of claim 3. For every $g \in \operatorname{Stab}(C)$ we have $g\left(C_{x}\right)=C_{x}$. Letting $z_{0}$ denote the isobarycentre of $K$ we consider an automorphism $\gamma \in \operatorname{Aut}\left(\mathbb{A}^{2}\right),(x, y) \mapsto\left(x, y-z_{0} x^{b}\right)$. It 
is easily seen that $\gamma$ preserves the pencil $\mathcal{L}$, while $\nu(\gamma): z \mapsto z-z_{0}$. Since $\nu\left(\gamma g \gamma^{-1}\right)=$ $\nu(\gamma) \nu(g) \nu(\gamma)^{-1}: 0 \mapsto 0$ we obtain $\gamma g \gamma^{-1}: C_{y} \rightarrow C_{y}$ and $C_{x} \rightarrow C_{x}$. Hence $\gamma \circ g \circ \gamma^{-1} \in \mathbb{T}$ (see corollary 2.10(b)) and so $\gamma \operatorname{Stab}(C) \gamma^{-1} \subseteq \mathbb{T}$.

However, for $C$ as in proposition 2.14 the stabilizer $\operatorname{Stab}(C)$ is not necessarily contained in $\mathrm{GL}(2, \mathbb{C})$, as is seen in the following exemple.

Example 2.15. Let $C=\left\{y\left(y-x^{b}\right)=0\right\}$, where $b>1$, and let $g:(x, y) \mapsto\left(x, x^{b}-y\right)$. Then $g \in \operatorname{Stab}(C) \backslash \mathrm{GL}(2, \mathbb{C})$.

From corollary 2.10 and propositions 2.12-2.14 we deduce the following result.

Corollary 2.16. The stabilizer $\operatorname{Stab}(C)$ of an acyclic plane curve $C$ is abelian unless $C$ is equivalent under the Aut $\left(\mathbb{A}^{2}\right)$-action to a union of affine lines through the origin.

\section{ACYCliC CURVES on AFFine tORIC surfaCeS}

In this section we classify acyclic curves on affine toric surfaces, similarly as this is done for plane acyclic curves in theorems 1.1 and 1.3.

3.1. Acyclic curves in the smooth locus. We start with the curves that do not pass through the singular point.

Let $d$ and $e$ be coprime integers with $0<e<d$, and let $\zeta \in \mathbb{C}^{\times}$be a primitive root of unity of degree $d$. Consider an affine toric surface $X_{d, e}=\mathbb{A}^{2} / G_{d, e}$, where $G_{d, e}=\langle g\rangle$ is the cyclic group generated by an element $g=\left(\begin{array}{ll}\zeta^{e} & 0 \\ 0 & \zeta\end{array}\right) \in \mathrm{GL}(2, \mathbb{C})$. Let $Q=\pi(\overline{0})$ denote the unique singular point of $X_{d, e}$, where $\pi: \mathbb{A}^{2} \rightarrow X_{d, e}$ is the quotient morphism and $\overline{0}=(0,0) \in \mathbb{A}^{2}$. We let $N\left(G_{d, e}\right)$ denote the normalizer of the subgroup $G_{d, e}$ in $\operatorname{GL}(2, \mathbb{C})$ and by $\mathcal{N}\left(G_{d, e}\right)$ that in the group $\operatorname{Aut}\left(\mathbb{A}^{2}\right)$.

Remark 3.1. By [16, Section 2.6], the surfaces $X_{d, e}$ and $X_{d^{\prime}, e^{\prime}}$ are isomorphic if and only if $d=d^{\prime}$ and either $e=e^{\prime}$ or $e e^{\prime}=1 \bmod d$. The latter two possibilities are related via the twist $\tau:(x, y) \mapsto(y, x)$ on $\mathbb{A}^{2}$.

For a linear form $l$ on $\mathbb{A}^{2}$ and for $c \in \mathbb{C}$ we let $C_{l}(c)$ denote the affine line $l=c$.

Theorem 3.2. (a) Up to the action of the automorphism group $\operatorname{Aut}\left(X_{d, e}\right)$, any irreducible acyclic curve $\mathcal{C}$ on $X_{d, e}$ which do not pass through the singular point $Q \in X_{d, e}$ is equivalent either to $\pi\left(C_{x}(1)\right)$ or to $\pi\left(C_{y}(1)\right)$. In particular, $\mathcal{C}$ coincides with an orbit of a $\mathbb{G}_{a}$-action on $X_{d, e}$, and also with an orbit closure of a $\mathbb{G}_{m}$-action on $X_{d, e}$.

(b) The curves $\pi\left(C_{x}(1)\right)$ and $\pi\left(C_{y}(1)\right)$ are equivalent on $X_{d, e}$ if and only if $e^{2} \equiv 1 \bmod d$.

Proof. To show (a) we let $\mathcal{C}$ be an irreducible acyclic curve on $X_{d, e}$ not passing through the singular point $Q$ and $C=\pi^{-1}(\mathcal{C})$ be its total preimage in $\mathbb{A}^{2}$. The morphism $\pi$ is finite of degree $d$, so any component $C^{i}$ of $C$ maps to $\mathcal{C}$ properly. Since the cyclic group $G_{d, e}$ acts freely on $\mathbb{A}^{2} \backslash\{\overline{0}\}$ and $C \subseteq \mathbb{A}^{2} \backslash\{\overline{0}\}$, the map $\left.\pi\right|_{C^{i}}: C^{i} \rightarrow \mathcal{C}$ is a non-ramified covering. However, $\mathcal{C}$ being simply connected it does not admit any non-trivial covering. Therefore $C$ has $d$ disjoint irreducible components $C^{1}, \ldots, C^{d}$ mapped isomorphically onto $\mathcal{C}$ under $\pi$. Furthermore, the cyclic group $G_{d, e}$ acts simply transitively on the set $\left\{C^{1}, \ldots, C^{d}\right\}$.

Write a reduced defining equation of $C^{1}$ as $p-1=0$, where $p \in \mathbb{C}[x, y]$. Any regular invertible function on a connected and simply connected variety is constant. Hence for every 
$i=1, \ldots, d$ the restriction of $p \mid C^{i}$ is constant, say, $\kappa_{i}$ i.e., $C^{i} \subseteq p^{-1}\left(\kappa_{i}\right)$. If $C^{1}$ were singular then by theorem 1.3 it would be equivalent to a curve $C_{a, b}=\left\{y^{a}-x^{b}=0\right\}$, where $\min \{a, b\}>$ 1. For $c \neq 0$ the Euler characteristic of the fiber $y^{a}-x^{b}=c$ is negative. Hence this fiber cannot carry a curve with Euler characteristic 1 . This leads to a contradiction, because $d>1$ by our assumption. Thus the curve $C^{1}$ is smooth.

It follows that every fiber of $p$ is isomorphic to $\mathbb{A}^{1}$. Hence there is an automorphism $\delta \in \operatorname{Aut}\left(\mathbb{A}^{2}\right)$ sending the curves $C^{i}$ to the lines $y=\kappa_{i}$ with distinct $k_{i}$, where $\kappa_{1}=1$. Moreover, we may suppose that $\delta(\overline{0})=\overline{0}$. Letting $g^{\prime}=\delta \circ g \circ \delta^{-1}$ we obtain $g^{\prime}(\overline{0})=\overline{0}$, $g^{\prime d}=g^{d}=\mathrm{id}$, and $g^{\prime}\left(C_{y}\right)=C_{y}$ i.e., $g^{\prime} \in \operatorname{Stab}\left(C_{y}\right)=\mathrm{Jonq}^{+}\left(\mathbb{A}^{2}\right)$, see proposition 2.8. Furthermore,

$$
\rho\left(g^{\prime}\right)=d g^{\prime}(\overline{0})=d \delta(\overline{0}) \circ g \circ(d \delta(\overline{0}))^{-1} \in \mathbb{T},
$$

where $\rho: \mathrm{Jonq}^{+}\left(\mathbb{A}^{2}\right) \rightarrow \mathbb{T}$ is the canonical surjection (see proposition 2.5). Hence the elements $g=\rho(g) \in \mathbb{T}$ and $\rho\left(g^{\prime}\right) \in \mathbb{T}$ are conjugated in $\mathrm{GL}(2, \mathbb{C})$ and so either $\rho\left(g^{\prime}\right)=\rho(g)=g$ or $\rho\left(g^{\prime}\right)=\tau g \tau$.

Since $\operatorname{ord}\left(g^{\prime}\right)=d>1$ we have $g^{\prime} \notin U^{+}$. It follows from lemma 2.1 and proposition 2.5 that $\mu^{-1} g^{\prime} \mu=\rho\left(g^{\prime}\right)$ for a suitable $\mu \in U^{+}$. In the case where $\rho\left(g^{\prime}\right)=g$ we obtain $\mu^{-1} \delta g \delta^{-1} \mu=g$, and in the case where $\rho\left(g^{\prime}\right)=\tau g \tau$ we get $\tau \mu^{-1} \delta g \delta^{-1} \mu \tau=g$. In the former case $\mu^{-1} \delta\left(C^{1}\right)=C_{y}(1)$, where $\mu^{-1} \delta \in \mathcal{N}\left(G_{d, e}\right)$, while in the latter one $\tau \mu^{-1} \delta\left(C^{1}\right)=C_{x}(1)$, where again $\tau \mu^{-1} \delta \in \mathcal{N}\left(G_{d, e}\right)$. Since the corresponding element normalizes the group $G_{d, e}$ it descends to an automorphism of the surface $X_{d, e}$ which sends $\mathcal{C}$ to the curve $\pi\left(C_{y}(1)\right)$ in the former case and to $\pi\left(C_{x}(1)\right)$ in the latter one.

Furthermore, $\mathcal{C}$ is an orbit of a $\mathbb{G}_{a}$-action on $X_{d, e}$ induced by a $\mathbb{G}_{a}$-action on $\mathbb{A}^{2}$ with $C^{1}$ as an orbit, which commutes with the $G_{d, e^{-a c t i o n}}$ and is defined via

$$
t .(x, y)=\left(x, y+t x^{e^{\prime}}\right) \quad \text { if } \quad \mathcal{e}=\pi\left(C_{x}(1)\right) \quad \text { and } \quad t .(x, y)=\left(x+t y^{e}, y\right) \quad \text { if } \quad \mathcal{C}=\pi\left(C_{y}(1)\right) \text {, }
$$

respectively, where $t \in \mathbb{G}_{a}$. This shows (a).

To show (b) we assume first that $e^{2} \equiv 1 \bmod d\left(\right.$ and so $e^{\prime}=e$ ). Then the involution $\tau:(x, y) \mapsto(y, x)$ normalizes the subgroup $G_{d, e}$ and induces an automorphism of $X_{d, e}$ which interchanges the curves $\pi\left(C_{x}(1)\right)$ and $\pi\left(C_{y}(1)\right)$ (and also $\pi\left(C_{x}\right)$ and $\pi\left(C_{y}\right)$ ).

Conversely, assume that there is an automorphism of $X_{d, e}$ which sends $\pi\left(C_{x}(1)\right)$ to $\pi\left(C_{y}(1)\right)$. It can be lifted to an automorphism of the Cox ring $\mathbb{C}[x, y]$ of the surface $X_{d, e}$. Hence there is an element $\gamma \in \mathcal{N}\left(G_{d, e}\right)$ which sends $C_{x}(1)$ to $C_{y}(1)$ and, moreover, sends the variable $y$ to $x$. By proposition 2.8(a) $\gamma$ has the form $(x, y) \longmapsto(\alpha y+f(x), x)$. A direct computation shows that

$$
\gamma^{-1} \circ g \circ \gamma:(x, y) \longmapsto\left(\zeta x+h(y), \zeta^{e} y\right),
$$

where $\gamma^{-1} \circ g \circ \gamma \in G_{d, e}$ because $\gamma \in \mathcal{N}\left(G_{d, e}\right)$. It follows that $h=0$ and $\left(\zeta^{e}\right)^{e}=\zeta$ and so $e^{2} \equiv 1 \bmod d$. Now the proof is completed.

Corollary 3.3. Every irreducible, acyclic curve $\mathcal{C}$ on $X_{d, e}$ not passing through the singular point $Q \in X_{d, e}$ belongs to a pencil $\mathcal{L}$ consisting of one-dimensional orbits of an effective $\mathbb{G}_{a}$-action on $X_{d, e}$ and a fixed point curve $\mathfrak{C}_{0}$ passing through $Q$. The members of $\mathcal{L}$ different from $\mathcal{C}_{0}$ are equivalent under the $\mathbb{G}_{m}$-action on $X_{d, e}$ induced by the $\mathbb{T}_{1,1}$-action on $\mathbb{A}^{2}$.

The union of several one-dimensional orbits of a $\mathbb{G}_{a}$-action on $X_{d, e}$ is a disconnected, simply connected curve. In fact every such curve arises in this way, as the reader can easily derive from the previous results. 
Corollary 3.4. Every disconnected, simply connected curve $\mathrm{C}$ on $X_{d, e}$ is equivalent under the $\operatorname{Aut}\left(X_{d, e}\right)$-action either to $\bigcup_{i=1}^{r} \pi\left(C_{x}\left(\kappa_{i}\right)\right)$, or to $\bigcup_{i=1}^{r} \pi\left(C_{y}\left(\kappa_{i}\right)\right)$, where $r \geq 2$ and $\kappa_{1}, \ldots, \kappa_{r} \in$ $\mathbb{A}^{1}$ are distinct.

3.2. Acyclic curves through the singular point. In this subsection we describe the acyclic curves on a singular affine toric surface $X_{d, e}(d>1)$ passing through the singular point $Q$. We let as before $C_{a, b}=\left\{y^{a}-x^{b}=0\right\}$, where $a, b \geq 1$ and $\operatorname{gcd}(a, b)=1$. In particular $C_{1,1}=\{x-y=0\}$. We keep the notation $C_{x}=\{x=0\}$ and $C_{y}=\{y=0\}$.

Due to the following theorem, the set of all equivalence classes of irreducible, acyclic curves on $X_{d, e}$ through $Q$ is countable. A similar fact in the smooth case $X_{1,1}=\mathbb{A}^{2}$ is well known, see theorem 1.3(a).

Theorem 3.5. (a) Up to the action of the automorphism group $\operatorname{Aut}\left(X_{d, e}\right)$, every irreducible acyclic curve $\mathcal{C}$ on $X_{d, e}$ passing through the singular point $Q \in X_{d, e}$ is equivalent to one of the curves $\pi\left(C_{a, b}\right), \pi\left(C_{x}\right)$, or $\pi\left(C_{y}\right)$.

(b) The curves $\pi\left(C_{x}\right)$ and $\pi\left(C_{y}\right)$ are equivalent on $X_{d, e}$ if and only if $e^{2} \equiv 1 \bmod d$, if and only if the twist $\tau:(x, y) \longmapsto(y, x)$ descends to an automorphism of the surface $X_{d, e}$.

Proof. (a) The curve $D=\pi^{*}(\mathcal{C}) \backslash\{\overline{0}\}$ is reduced and the projection $D \rightarrow \mathcal{C} \backslash\{Q\}$ is an unramified cyclic covering of degree $d$. It follows that the irreducible components $D^{1}, \ldots, D^{r}$ of $D$ are disjoint, and for every $i=1, \ldots, r$ the restriction $\left.\pi\right|_{D^{i}}: D^{i} \rightarrow \mathcal{C} \backslash\{Q\}$ is an unramified cyclic covering. Furthermore, the cyclic group $G_{d, e}$ acts transitively on the set of these components i.e., the generator $g$ of $G_{d, e}$ permutes them cyclically. In particular, $r$ divides $d$ and $D^{i} \simeq \mathbb{A}_{*}^{1} \forall i$, while the closures $C^{i}=D^{i} \cup\{\overline{0}\}$ and $C=D \cup\{\overline{0}\}=\pi^{-1}(\mathcal{C})$ are acyclic. Clearly, the curve $C$ cannot have more than one singular point, hence it cannot be of type (III). According to the remaining types we distinguish the following cases.

Case 1: $r=1$ and $C=C^{1}$ is a smooth acyclic curve of type (I). Then $C^{1}$ is $G_{d, e^{-}}$ stable and passes through the origin. Similarly as in the proof of theorem 3.2, one can show that in suitable new coordinates in $\mathbb{A}^{2}$ we have $C^{1}=C_{y}$ and $g$ acts diagonally either via $(x, y) \mapsto\left(\zeta^{e} x, \zeta y\right)$, or via $(x, y) \mapsto\left(\zeta^{e^{\prime}} x, \zeta y\right)$. In the second case after transposition $(x, y) \mapsto(y, x)$ we obtain that $C^{1}=C_{x}$ and $g$ acts via $(x, y) \mapsto\left(\zeta x, \zeta^{e^{\prime}} y\right)$, that is by an element of the cyclic group $G_{d, e}$. In any case up to the $\operatorname{Aut}\left(X_{d, e}\right)$-action, the curve $\mathcal{C}$ is equivalent either to $\pi\left(C_{x}\right)$ or to $\pi\left(C_{y}\right)$.

Case 2: $r>1$ and $C$ is an acyclic curve of type (II) or (IV) with an ordinary singularity at the origin. By theorem 1.3 a suitable automorphism $\gamma \in \operatorname{Aut}\left(\mathbb{A}^{2}\right)$ sends the reduced curve $C=\pi^{*}(\mathcal{C})$ to a union $C^{\prime}=C^{\prime 1}+\ldots+C^{\prime r}$ of affine lines through the origin given by equation

$$
y\left(y-\kappa_{2} x\right) \ldots\left(y-\kappa_{r} x\right)=0, \quad \text { where } \kappa_{i} \in \mathbb{C}^{\times} \text {are distinct . }
$$

The curve $C^{\prime}$ is stable under the action on $\mathbb{A}^{2}$ of the cyclic group $\gamma G_{d, e} \gamma^{-1}=\left\langle g^{\prime}\right\rangle$, where $g^{\prime}=\gamma g \gamma^{-1} \in \operatorname{Aut}\left(\mathbb{A}^{2}\right)$. By corollary $2.9 \operatorname{Stab}\left(C^{\prime}\right) \subseteq \mathrm{GL}(2, \mathbb{C})$, hence $g^{\prime} \in \mathrm{GL}(2, \mathbb{C})$.

There exists an element $\delta \in \mathrm{GL}(2, \mathbb{C})$ such that $\delta g^{\prime} \delta^{-1}=g$ is diagonal and acts via $(x, y) \mapsto\left(\zeta^{e} x, \zeta y\right)$. Since no component $C^{i}$ of $C$ is stable under $g$, the composition $\delta \gamma$ sends each $C^{i}$ to a line through the origin different from a coordinate axis. Since all such lines are $\mathbb{T}$-equivalent, their images in the surface $X_{d, e}$ are also equivalent under the action on $X_{d, e}$ of the quotient torus $\mathbb{T}^{\prime}=\mathbb{T} / G_{d, e}$. 
The resulting automorphism $\delta \gamma$ from the centralizer of the subgroup $G_{d, e}$ in $\operatorname{Aut}\left(\mathbb{A}^{2}\right)$ rectifies $C$ and sends $C^{1}$ to a line $\mathbb{T}$-equivalent to $C_{1,1}$. Consequently, the curve $\mathcal{C}$ on $X_{d, e}$ is equivalent to $\pi\left(C_{1,1}\right)$ under the $\mathbb{T}^{\prime}$-action on $X_{d, e}$ and the automorphism $\pi_{*}(\delta \gamma) \in \operatorname{Aut}\left(X_{d, e}\right)$.

Case 3: $r>1$ and $C$ is an acyclic curve of type $(V)$ with smooth components $C^{i}$ and a non-ordinary singularity at the origin. By theorem 1.3 in this case a suitable automorphism $\gamma \in \operatorname{Aut}\left(\mathbb{A}^{2}\right)$ sends the reduced curve $C=C^{1}+\ldots+C^{r}$ on $\mathbb{A}^{2}$ to a curve $C^{\prime}$ given by equation

$$
x^{\varepsilon_{x}} y\left(y-\kappa_{2} x^{b}\right) \ldots\left(y-\kappa_{r} x^{b}\right)=0 \quad \text { with distinct } \kappa_{i} \in \mathbb{C}^{\times},
$$

where $b>1$ and $\varepsilon_{x} \in\{0,1\}$. The group $G_{d, e}^{\prime}=\gamma G_{d, e} \gamma^{-1} \subseteq \operatorname{Aut}\left(\mathbb{A}^{2}\right)$ acts transitively on the set of components $C^{\prime i}$ of $C^{\prime}$. Since the $G_{d, e^{\prime}}^{\prime}$ action preserves tangency we have $\varepsilon_{x}=0$. We may also suppose that $k_{2}=1$. Since $G_{d, e}^{\prime} \subseteq \operatorname{Stab}\left(C^{\prime}\right)$, the singular point $\overline{0} \in C^{\prime}$ is fixed under the $G_{d, e^{\prime}}^{\prime}$ action on $\mathbb{A}^{2}$.

There is an element $g^{\prime} \in G_{d, e}^{\prime}$ which sends the component $C^{\prime 2}=\left\{y-x^{b}=0\right\}$ to $C^{\prime 1}=C_{y}$. Since $h:(x, y) \mapsto\left(x, y-x^{b}\right)$ does the same, according to proposition 2.8(a) $g^{\prime}$ can be written as

$$
g^{\prime}:(x, y) \longmapsto\left(\alpha x+f\left(y-x^{b}\right), \beta\left(y-x^{b}\right)\right)
$$

for some $\alpha, \beta \in \mathbb{C}^{\times}$and $f \in \mathbb{C}[z]$. Hence $g^{\prime}$ maps the affine line $C^{\prime 1}=C_{y}$ to the parameterized curve $\left\{\left(\alpha t+f\left(-t^{b}\right),-\beta t^{b}\right) \mid t \in \mathbb{A}^{1}\right\}$. However $g^{\prime}\left(C^{\prime 1}\right)=C^{\prime i}$ for some $i \in\{2, \ldots, r\}$. It follows that $f=0$. Therefore $g^{\prime}:(x, y) \mapsto\left(\alpha x, \beta\left(y-x^{b}\right)\right)$ and so

$$
g^{\prime k}:(x, y) \mapsto\left(\alpha^{k} x, \beta^{k} y-\beta x^{b}\left(\beta^{k-1}+\alpha^{b} \beta^{k-2}+\ldots+\alpha^{b(k-1)}\right)\right) .
$$

Since $g^{\prime k}=$ id for some $k \mid d$, we have $\alpha^{k}=\beta^{k}=1$ and $\left(\beta / \alpha^{b}\right)^{k-1}+\ldots+1=0$. This implies that $\beta \neq \alpha^{b}$.

Thus the triangular automorphism

$$
h^{\prime}:(x, y) \mapsto\left(x, y+\frac{\beta}{\alpha^{b}-\beta} x^{b}\right)
$$

is well defined and sends the curve $C^{\prime}$ to a new one $C^{\prime \prime}=\sum_{i} C^{\prime \prime i}$ given by a similar equation. Furthermore, $h^{\prime}$ conjugates $g^{\prime}$ with

$$
g^{\prime \prime}:(x, y) \longmapsto(\alpha x, \beta y),
$$

where $(\alpha, \beta)$ can be written either as $\left(\xi^{e}, \xi\right)$ or as $\left(\xi^{e^{\prime}}, \xi\right)$ for a primitive $d$ th root of unity $\xi\left(\right.$ recall that $\left.e e^{\prime} \equiv 1 \bmod d\right)$. In the latter case we apply additionally the transposition of coordinates to get $(\alpha, \beta)=\left(\xi, \xi^{e^{\prime}}\right)$. The composition $h^{\prime} \gamma$ normalizes the group $G_{d, e}$ in $\operatorname{Aut}\left(\mathbb{A}^{2}\right)$ and sends $C^{1}$ to a member of the pencil $\mathcal{L}$, where as before

$$
\mathcal{L}=\left\{y-\kappa x^{b}=0\right\}_{\kappa \in \mathbb{C}} \cup\left\{b C_{x}\right\} .
$$

However, every member of $\mathcal{L}$ different from the coordinate axes $C_{x}, C_{y}$ is equivalent to the curve $C_{1, b}$ under the $\mathbb{T}$-action on $\mathbb{A}^{2}$. Finally $h^{\prime} \gamma$ induces an automorphism $\theta$ of the quotient surface $X_{d, e}$ such that the image under $\theta$ of the curve $\mathcal{C}=\pi\left(C^{1}\right)$ is $\mathbb{T}^{\prime}$-equivalent either to $\pi\left(C_{y}\right)$ or to $\pi\left(C_{1, b}\right)$, as required.

Case 4: $C$ is an acyclic curve of type (VI) with all the components $C^{i}$ being singular. By theorem 1.3 a suitable automorphism $\gamma \in \operatorname{Aut}\left(\mathbb{A}^{2}\right)$ sends $C$ to a curve $C^{\prime}$ given by equation (2), where $\varepsilon_{x}=\varepsilon_{y}=0, a, b>1$, and $\operatorname{gcd}(a, b)=1$. We may also assume that $\kappa_{1}=1$ and so 
$C^{1}=C_{a, b}$. Let as before $g^{\prime}=\gamma g \gamma^{-1}$. Since the $\mathbb{T}$-action is transitive on the members of the pencil

$$
\mathcal{L}=\left\{y^{a}-\kappa x^{b}=0\right\}_{\kappa \in \mathbb{C}} \cup\left\{b C_{x}\right\}
$$

different from the coordinate axes $C_{x}$ and $C_{y}$, there is an element $h \in \mathbb{T}$ such that $g^{\prime \prime}=h \circ g^{\prime}$ preserves the curve $C_{a, b}$. By proposition $2.12 g^{\prime \prime} \in \mathbb{T}$, hence also $g^{\prime} \in \mathbb{T}$. Since the eigenvalues $\zeta^{e}$ and $\zeta$ of $g$ and $g^{\prime}$ are the same, up to interchanging the coordinates we have $g^{\prime} \in G_{d, e}$. Hence $\gamma$ normalizes the group $G_{d, e}$. Reasoning as before we conclude that the curve $\mathcal{C}$ on the surface $X_{d, e}$ is equivalent to $\pi\left(C_{a, b}\right)$, as stated. This shows (a). The proof of (b) goes in the same way as that of theorem $3.2(\mathrm{~b})$ and so we leave it to the reader.

Remarks 3.6. 1 . If $e>1$ then the curve $\pi^{-1}\left(\pi\left(C_{x}\right)\right)=C_{x}$ on $\mathbb{A}^{2}$ is irreducible, while $\pi^{-1}\left(\pi\left(C_{1,1}\right)\right)$ is reducible. Hence these plane curves are not equivalent and so their images $\pi\left(C_{x}\right)$ and $\pi\left(C_{1,1}\right)$ on the surface $X_{d, e}$ are not equivalent either (under the $\operatorname{Aut}\left(X_{d, e}\right)$-action).

2. The following simple example shows that, in contrast with theorem 3.2, a curve on $X_{d, e}$ isomorphic to the affine line and passing through the singular point can be non-equivalent to the image of one of the curves $C_{1,1}, C_{x}$, and $C_{y}$.

Indeed, the curve $C_{2,3}$ in $\mathbb{A}^{2}$ is singular. Nonetheless, its image $\mathcal{C}$ in the affine toric surface $X_{5,4}$ passes through the singular point and is isomorphic to the affine line $\mathbb{A}^{1}$. Clearly, $\mathcal{C}$ is not equivalent in $X_{5,4}$ to $\pi\left(C_{1,1}\right), \pi\left(C_{x}\right)$, or $\pi\left(C_{y}\right)$.

3.3. Acyclic curves as orbit closures. Summarizing the results of the previous subsections we arrive at the following alternative description.

Theorem 3.7. Let $X$ be an affine toric surface over $\mathbb{C}$ with the acting torus $T$. Then every irreducible acyclic curve $\mathcal{C}$ on $X$ coincides with the closure of a non-closed orbit of a regular $\mathbb{G}_{m}$-action on $X .{ }^{4}$ Furthermore, up to an automorphism of $X$, such a curve $\mathcal{C}$ is the closure of a non-closed orbit of a subtorus of $T$.

Proof. Let $\mathcal{C}$ be an irreducible acyclic curve on a toric surface $X$. If $X$ is smooth then this is one of the surfaces $\mathbb{A}_{*}^{1} \times \mathbb{A}_{*}^{1}, \mathbb{A}^{1} \times \mathbb{A}_{*}^{1}$, or $\mathbb{A}^{2}$. There is no acyclic curve on $X=\mathbb{A}_{*}^{1} \times \mathbb{A}_{*}^{1}$, and the only irreducible acyclic curves on $\mathbb{A}^{1} \times \mathbb{A}_{*}^{1}$ are of the form $\mathbb{A}^{1} \times\{\mathrm{pt}\}$. Hence our assertion holds for these surfaces. In the case of the affine plane $X \simeq \mathbb{A}^{2}$ the result follows from theorems 1.1 and 1.3.

By $[16, \S 2.2]$, every singular affine toric surface is isomorphic to one of the surfaces $X_{d, e}=$ $\mathbb{A}^{2} / G_{d, e}$, where $d>1$. Finally in this case the result follows from theorems 3.2 and 3.5.

Corollary 3.8. Any irreducible acyclic curve on an affine toric surface has at most one singular point. If the surface is singular then this point coincides with the singular point of the surface.

The following result generalizes theorem 1.1 of Abhyankar, Moh, and Suzuki.

Theorem 3.9. Up to the action of the group $\operatorname{Aut}\left(X_{d, e}\right)$ there are only finitely many different embeddings $\mathbb{A}^{1} \hookrightarrow X_{d, e}$.

Proof. It suffices to show that the smooth curves on $X_{d, e}$ of the form $\pi\left(C_{a, b}\right)$ belong to a finite set of equivalence classes. Notice that the subalgebra $\mathbb{C}[x, y]^{G_{d, e}}$ of $G_{d, e^{-i n v a r i a n t s}}$ is generated by the monomials $y^{d}, x y^{c_{1}}, \ldots, x^{d-1} y^{c_{d-1}}, x^{d}$, where $0<c_{k}<d$ and $c_{k}+k e \equiv 0$

\footnotetext{
${ }^{4}$ Clearly, such an orbit closure is acyclic.
} 
mod $d$. These monomials define a closed embedding $X_{d, e} \hookrightarrow \mathbb{A}^{d+1}$. The image of the curve $\pi\left(C_{a, b}\right)$ under this embedding is

$$
\left(t^{d b}, t^{a+c_{1} b}, \ldots, t^{(d-1) a+c_{d-1} b}, t^{d a}\right), \quad t \in \mathbb{C} .
$$

This image is smooth if and only if one of the exponents, say, $\delta$ of our monomials coincides with the greatest common divisor of all the exponents. Since $a$ and $b$ are coprime $\delta \mid d$.

In the case where $\delta=k a+c_{k} b$ for some $k \geq 1$ we obtain $\delta=k a+c_{k} b \leq d$ and so $a+b \leq d$. The number of all possible such pairs $(a, b)$ is finite.

If $\delta \in\{d a, d b\}$ then $a=1$ or $b=1$ because $\operatorname{gcd}(a, b)=1$. Suppose for instance that $a=1$ and $\delta=d$, the other case being similar. Thus

$$
e k+c_{k} \equiv 0 \quad \bmod d \text { and } k+b c_{k} \equiv 0 \bmod d
$$

for all possible values of $k \geq 1$. For $k=1$ it follows that $e b \equiv 1 \bmod d$. Hence the curve $C_{a, b}=C_{1, b}$ is stable under the $G_{d, e^{-}}$action on $\mathbb{A}^{2}$. The automorphism

$$
(x, y) \longmapsto\left(x, y-x^{b}\right)
$$

commutes with the $G_{d, e^{-}}$action and sends this curve to the axis $C_{y}$. Therefore for any $b \geq 1$ the curves $\pi\left(C_{1, b}\right)$ and $\pi\left(C_{y}\right)$ on $X_{d, e}$ are equivalent. Now the proof is completed.

Remark 3.10. Consider an affine toric variety $X$ of dimension $n$. It is known (see [8]) that if an $(n-1)$-dimensional torus $T^{\prime}$ acts effectively on $X$, then $T^{\prime}$ is conjugate in the group $\operatorname{Aut}(X)$ to a subtorus of the acting torus $T$ of $X$. An analogous result for tori of codimension $\geq 2$ is unknown. We conclude section 3 by the following related problem.

Questions. Let $X$ be an affine toric variety of dimension $n$ with acting torus $T$. Assume that a closed subvariety $Y$ of $X$ admits in turn an action of an algebraic torus $T^{\prime}$ with an open orbit.

Is it true that $Y$ can be realized as the orbit closure of a $T^{\prime}$-action on $X$ ? Is, moreover, this $T^{\prime}$-action on $X$ conjugated to the action on $X$ of a subtorus of the acting torus $T$ ?

This is indeed the case for $n=2$ as follows from theorem 3.7.

3.4. Reducible acyclic curves on affine toric surfaces. Let us start with the following lemma.

Lemma 3.11. Consider the affine toric surface $X=X_{d, e}$ with the quotient map $\pi: \mathbb{A}^{2} \rightarrow$ $X=\mathbb{A}^{2} / G_{d, e}$. If $\mathcal{C}$ is a reduced, simply connected curve on $X$, then the total transform $C=\pi^{*}(\mathrm{C})$ of $\mathrm{C}$ in $\mathbb{A}^{2}$ is also reduced and simply connected.

Proof. If $d=1$ i.e., $X \simeq \mathbb{A}^{2}$, or the curve $\mathcal{C}$ is irreducible, then the assertion follows by the same argument as in the proof of theorems 3.2 and 3.5. Assume further that $d>1$ and $\mathcal{C}$ is reducible. Letting $Q=\pi(\overline{0}) \in X$ and $\pi^{*}(\mathcal{C})=C=C^{1}+\ldots+C^{s}$, where every irreducible component $C^{i}$ of $C$ is simply connected, we consider the following cases.

Case 1: Every irreducible component $\mathrm{C}^{k}$ of $\mathcal{C}$ passes through $Q$. Then any two such components meet only at $Q$, and it is easily seen that any two distinct components $C^{i}$ and $C^{j}$ of $C$ also meet only at the origin. In this case $C$ is connected and simply connected i.e., acyclic.

Case 2: There are two distinct crossing components, say, $\mathrm{e}^{k}$ and $\mathrm{e}^{l}$ of $\mathcal{C}$ not passing through Q. According to theorem 3.2 (cf. also Corollaries 3.3 and 3.4), under the action of the 
normalizer $\mathcal{N}\left(G_{d, e}\right)$ on $\mathbb{A}^{2}$ the total transform $\pi^{*}\left(\complement^{k}\right)$ (or $\left.\pi^{*}\left(\complement^{l}\right)\right)$ is equivalent to a union of $d$ parallel lines, which are parallel either to $C_{x}$ or to $C_{y}$ and are cyclically permuted under the $G_{d, e^{-a c t i o n}}$ Furthermore, each component of $\pi^{*}\left(\mathrm{C}^{k}\right)$ meets every component of $\pi^{*}\left(\mathrm{e}^{l}\right)$ in $d$ distinct points. All these $d^{2}$ intersection points must project to the unique intersection point $\mathrm{e}^{k} \cap \mathrm{e}^{l}$. Hence they should belong to a $G_{d, e^{-}}$orbit, which is impossible. This contradiction shows that the components of $\mathcal{C}$ not passing through $Q$ do not meet.

Case 3: There is just one component, say, $\mathcal{C}^{1}$ of $\mathcal{C}$ not passing through $Q$. The union of the other components of $\mathcal{C}$ can meet $\mathcal{C}^{1}$ in at most one point, and they meet each other at $Q$. Hence either $\mathcal{C}^{1}$ does not meet this union, or there is just one component, say, $\mathcal{C}^{2}$ of $\mathcal{C}$ passing through $Q$ which meets $\mathcal{C}^{1}$. In the former case the reduced curve $C=\pi^{*}(\mathcal{C})$ is clearly simply connected, as stated. In the latter case we may assume as before that $\pi^{*}\left(\mathrm{C}^{1}\right)$ is a union of $d$ lines parallel to a coordinate axis and cyclically permuted under the $\mathbb{G}_{d, e}$-action. Every component of the total transform $\pi^{*}\left(\mathfrak{C}^{2}\right)$ is simply connected, passes through the origin, and meets one of these lines. Hence it meets all the parallel lines. If the curve $\pi^{*}\left(\mathfrak{C}^{2}\right)$ is reducible then its irreducible components meet only at the origin, and meet one of the lines in $\pi^{*}\left(\mathrm{C}^{1}\right)$ in at least two distinct points. These points project in $X$ to distinct smooth points. The latter contradicts the assumption of simply connectedness of the curve $\mathcal{C}$, because in this case we obtain a non-contractible cycle in $\mathcal{C}$. Hence the curve $\pi^{*}\left(\mathfrak{C}^{2}\right)$ is irreducible and meets every line in $\pi^{*}\left(\mathfrak{C}^{1}\right)$ in just one point, while the other curves $\pi^{*}\left(\mathfrak{C}^{k}\right), k \geq 3$, do not meet these lines at all. This shows that $C$ is simply connected.

Case 4: There are two disjoint components, say, $\mathrm{C}^{1}$ and $\mathrm{C}^{2}$ of $\mathcal{C}$ not passing through $Q$. Since the total transforms $\pi^{*}\left(\mathrm{C}^{1}\right)$ and $\pi^{*}\left(\mathrm{C}^{2}\right)$ are disjoint, they can be simultaneously transformed into unions of lines parallel to the same coordinate axis. Hence every component, say, $\mathcal{C}^{3}$ of $\mathcal{C}$ passing through $Q$ and meeting $\mathrm{C}^{1}$ meets also $\mathfrak{C}^{2}$, and vice versa. Using the same argument as before it is easily seen that there could be at most one such component $\mathfrak{C}^{3}$, and the total preimage $C=\pi^{*}(\mathcal{C})$ is simply connected, as required.

This ends the proof.

Using this lemma, in the following theorem we give a description of all reduced, simply connected curves on affine toric surfaces.

Theorem 3.12. Every reduced, acyclic curve $\mathcal{C}$ on $X=X_{d, e}$ is equivalent to a curve $\pi(C)$, where $C \subseteq \mathbb{A}^{2}$ is given by one of the equations

$$
x \prod_{i=1}^{r}\left(y^{a}-\kappa_{i} x^{b}\right)=0 \quad \text { or } \quad y \prod_{i=1}^{r}\left(x^{a}-\kappa_{i} y^{b}\right)=0, \quad \text { where } \quad b \geq 0, \quad a \geq 1, \quad \operatorname{gcd}(a, b)=1
$$

and where $\kappa_{i} \in \mathbb{C}(i=1, \ldots, r)$ are pairwise distinct.

Proof. Indeed, by lemma 3.11 the reduced plane curve $C=\pi^{*}(\mathcal{C})$ is simply connected. However, by theorem 1.3 every reduced, acyclic curve in $\mathbb{A}^{2}$ is given in appropriate coordinates by equation (18), while every reduced, disconnected, simply connected affine plane curve is equivalent to a finite union of parallel lines. It remains to show that, up to a permutation of the symbols $x$ and $y$, the corresponding coordinate change can be chosen in the normalizer $\mathcal{N}\left(G_{d, e}\right)$ of the group $G_{d, e}$ in $\operatorname{Aut}\left(\mathbb{A}^{2}\right)$. The latter can be done in the same way as in the proof of theorems 3.2 and 3.5. We leave the details to the reader. 


\section{Automorphism groups of AfFine toric surfaces}

In this section we prove an analog of the Jung-van der Kulk theorem 1.4 for affine toric surfaces and study algebraic groups acting on such surfaces.

4.1. Free amalgamated product structure. Consider again an affine toric surface $X_{d, e}=$ $\mathbb{A}^{2} / G_{d, e}$. We assume as usual that $1 \leq e<d, \operatorname{gcd}(d, e)=1$, and $G_{d, e}=\langle g\rangle$, where

$$
g:(x, y) \longmapsto\left(\zeta^{e} x, \zeta y\right) \quad \text { with } \quad \zeta=\exp \left(\frac{2 \pi i}{d}\right) .
$$

Notation 4.1. Let $G \subseteq \mathrm{GL}(2, \mathbb{C})$. Letting as before $N(G)$ denote the normalizer of $G$ in the group $\operatorname{GL}(2, \mathbb{C})$ and $\mathcal{N}(G)$ that in the group $\operatorname{Aut}\left(\mathbb{A}^{2}\right)$, we abbreviate

$$
N_{d, e}=N\left(G_{d, e}\right) \text { and } \mathcal{N}_{d, e}=\mathcal{N}\left(G_{d, e}\right) .
$$

It is easily seen that

$$
N_{d, e}= \begin{cases}\mathrm{GL}(2, \mathbb{C}) & \text { if } e=1, \\ N(\mathbb{T})=\langle\mathbb{T}, \tau\rangle & \text { if } e>1 \text { and } e^{2} \equiv 1 \bmod d, \\ \mathbb{T} & \text { otherwise }\end{cases}
$$

where $\tau:(x, y) \longmapsto(y, x)$ is a twist and $\mathbb{T}$ stands for the maximal torus in $\operatorname{GL}(2, \mathbb{C})$ consisting of the diagonal matrices. We let $B^{ \pm}$denote the Borel subgroup of all upper (lower, respectively) triangular matrices in $\mathrm{GL}(2, \mathbb{C})$. Consider the subgroups

$$
N_{d, e}^{ \pm}=N_{d, e} \cap \operatorname{Jonq}^{ \pm}\left(\mathbb{A}^{2}\right)= \begin{cases}B^{ \pm} & \text {if } e=1, \\ \mathbb{T} & \text { otherwise }\end{cases}
$$

and

$$
\mathcal{N}_{d, e}^{ \pm}=\mathcal{N}_{d, e} \cap \operatorname{Jonq}^{ \pm}\left(\mathbb{A}^{2}\right)
$$

The latter subgroups are described in lemma 4.5 below. Notice that

$$
\mathcal{N}_{d, e}^{+} \cap \mathcal{N}_{d, e}^{-}=\operatorname{Jonq}^{+}\left(\mathbb{A}^{2}\right) \cap \operatorname{Jonq}^{-}\left(\mathbb{A}^{2}\right)=\mathbb{T} .
$$

With this notation we can state an analog of theorem 1.4 by Jung and van der Kulk.

Theorem 4.2. If $e^{2} \not \equiv 1 \bmod d$ then

$$
\operatorname{Aut}\left(X_{d, e}\right) \simeq \mathcal{N}_{d, e}^{+} / G_{d, e} * \mathbb{T} / G_{d, e} \mathcal{N}_{d, e}^{-} / G_{d, e},
$$

while for $e^{2} \equiv 1 \bmod d$ we have

$$
\operatorname{Aut}\left(X_{d, e}\right) \simeq \mathcal{N}_{d, e}^{+} / G_{d, e} *_{N_{d, e}^{+} / G_{d, e}} N_{d, e} / G_{d, e} .
$$

There should be possible to derive this theorem by using the techniques elaborated by Danilov and Gizatullin [18]. However, we prefer a direct approach through an equivariant version 4.8 of the Abhyankar-Moh-Suzuki theorem. Within this approach theorem 4.2 is an immediate consequence of lemma 4.3 and proposition 4.4 below.

Lemma 4.3. There is an isomorphism

$$
\operatorname{Aut}\left(X_{d, e}\right) \simeq \mathcal{N}_{d, e} / G_{d, e} .
$$


Proof. The affine plane $\mathbb{A}^{2}$ can be viewed as the spectrum of a Cox ring of the toric surface $X_{d, e}$, see [13] or [3, I.6.1]. Hence every automorphism $\Phi \in \operatorname{Aut}\left(X_{d, e}\right)$ can be lifted (in a non-unique way) to an element $\varphi \in \mathcal{N}_{d, e} .{ }^{5}$ This yields an exact sequence (see [5, Thm. 5.1])

$$
1 \rightarrow G_{d, e} \rightarrow \mathcal{N}_{d, e} \rightarrow \operatorname{Aut}\left(X_{d, e}\right) \rightarrow 1,
$$

as claimed.

Proposition 4.4. If $e^{2} \not \equiv 1 \bmod d$ then

$$
\mathcal{N}_{d, e} \simeq \mathcal{N}_{d, e}^{+} *_{\mathbb{T}} \mathcal{N}_{d, e}^{-},
$$

while for $e^{2} \equiv 1 \bmod d$

$$
\mathcal{N}_{d, e} \simeq \mathcal{N}_{d, e}^{+} *_{N_{d, e}^{+}} N_{d, e} .
$$

The proof is done in lemmas 4.5-4.14 below.

Theorem 4.2 follows now from lemma 4.3 and proposition 4.4 due to the fact that the subgroup $G_{d, e}$ is normal in every group that participates in (23) and (24). Indeed, this can be seen directly or, alternatively, derived as a simple consequence of a theorem by Cohen [11] on preservation of the free amalgamated product structure in the quotient.

Recall (see remark 2.2) that the polynomial ring $A=\mathbb{C}[t]$ possesses a $\mathbb{Z} / d \mathbb{Z}$-grading

$$
A=\bigoplus_{i=0}^{d-1} A_{d, i}, \quad \text { where } \quad A_{d, i}=t^{i} \mathbb{C}\left[t^{d}\right] .
$$

In terms of this grading the normalizer $\mathcal{N}_{d, e}^{ \pm}$admits the following description.

Lemma 4.5. (a) The group $\mathcal{N}_{d, e}^{+}\left(\mathcal{N}_{d, e}^{-}\right.$, respectively) consists of all de Jonqières transformations $\varphi^{+}$as in (7) $\left(\varphi^{-}\right.$as in (8), respectively) with $f \in A_{d, e}\left(f \in A_{d, e^{\prime}}\right.$, respectively).

(b) The subgroup $\mathcal{N}_{d, e}^{ \pm}$is the centralizer of $G_{d, e}$ in the group $\mathrm{Jonq}^{ \pm}\left(\mathbb{A}^{2}\right)$.

Proof. We stick to the plus-case, the proof in the other one being similar. We have

$$
\varphi^{+} \circ g \circ\left(\varphi^{+}\right)^{-1}:(x, y) \longmapsto\left(\zeta^{e} x+f\left(\zeta \frac{y}{\beta}\right)-\zeta^{e} f\left(\frac{y}{\beta}\right), \zeta y\right) .
$$

Hence $\varphi^{+} \circ g \circ\left(\varphi^{+}\right)^{-1} \in G_{d, e}$ if and only if $f(\zeta t)=\zeta^{e} f(t)$, if and only if $f \in A_{d, e}$. This shows (a). In the latter case $\varphi^{+} \circ g \circ\left(\varphi^{+}\right)^{-1}=g$, so (b) follows.

For a pair of polynomials $\varphi=(u, v)$ we $\operatorname{let} \operatorname{deg} \varphi=\max \{\operatorname{deg} u, \operatorname{deg} v\}$. The following result is an immediate consequence of lemma 4.5.

Lemma 4.6. Assume as before that $1 \leq e<d$ and $\operatorname{gcd}(d, e)=1$. Then the following hold.

(a) $\varphi(\overline{0})=\overline{0} \forall \varphi \in \mathcal{N}_{d, e}^{ \pm}$.

(b) $\mathcal{N}_{d, e}^{ \pm} \cap \operatorname{Aff}\left(\mathbb{A}^{2}\right)=N_{d, e}^{ \pm}= \begin{cases}B^{ \pm}, & e=1, \\ \mathbb{T}, & e>1 .\end{cases}$

(c) Let $\varphi^{ \pm}$be as in (7) and (8), respectively. Assume that $\varphi^{ \pm} \notin \mathbb{T}$. Then $\operatorname{deg} \varphi^{+} \geq e$ and $\operatorname{deg} \varphi^{-} \geq e^{\prime}$, where $1 \leq e^{\prime}<d$ and $e e^{\prime} \equiv 1 \bmod d$.

\footnotetext{
${ }^{5}$ Alternatively, this follows from the monodromy theorem, see the proof of theorem 5.3 below.
} 
The next two lemmas provide a $G_{d, e^{-}}$equivariant version of the Abhyankar-Moh-Suzuki theorem 1.1.

Lemma 4.7. Let $C$ be a smooth, polynomial curve in $\mathbb{A}^{2}$ parameterized via $t \longmapsto(u(t), v(t))$, where $u, v \in t \mathbb{C}[t]$. If $G_{d, e} \subseteq \operatorname{Stab}(C)$ then either $(u, v) \in A_{d, e} \times A_{d, 1}$ or $(u, v) \in A_{d, 1} \times A_{d, e^{\prime}}$.

Proof. The tangent vector to $C$ at the origin is $w=\left(u^{\prime}(0), v^{\prime}(0)\right) \in \mathbb{A}^{2}$. Since it is stable under the tangent $G_{d, e^{-a c t i o n}}$ then either $g(w)=\zeta^{e} w$ or $g(w)=\zeta w$. Thus $g \mid C: C \rightarrow C$ acts either via $t \longmapsto \zeta^{e} t$ or via $t \longmapsto \zeta t$. In the former case

$$
g \circ(u, v)(t)=\left(\zeta^{e} u(t), \zeta v(t)\right)=\left(u\left(\zeta^{e} t\right), v\left(\zeta^{e} t\right)\right),
$$

and in the latter one

$$
g \circ(u, v)(t)=\left(\zeta^{e} u(t), \zeta v(t)\right)=(u(\zeta t), v(\zeta t)) .
$$

Now the assertion follows.

Lemma 4.8. For a curve $C$ as in lemma 4.7 there is an automorphism $\varphi \in\left\langle\mathcal{N}_{d, e}^{+}, \mathcal{N}_{d, e}^{-}\right\rangle$ which sends $C$ to one of the coordinate axes $C_{x}$ and $C_{y}$.

Proof. If $u=0$ or $v=0$ there is nothing to prove. Thus we may suppose that $\operatorname{deg} u \geq \operatorname{deg} v>$ 0 . By the Abhyankar-Moh-Suzuki theorem 1.1 we have $\operatorname{deg} u=n \operatorname{deg} v$ for some $n \in \mathbb{N}$. By virtue of lemma 4.7 either $(\operatorname{deg} u, \operatorname{deg} v) \equiv(e, 1) \bmod d$ or $(\operatorname{deg} u, \operatorname{deg} v) \equiv\left(1, e^{\prime}\right) \bmod d . \operatorname{In}$ both cases it follows that $n \equiv e \bmod d$ and so $\varphi_{1} \in \mathcal{N}_{d, e}^{+}$, where $\varphi_{1}:(x, y) \longmapsto\left(x-c y^{n}, y\right)$ (see lemma 4.5(a)). We have $\varphi_{1}(u, v)=\left(u_{1}, v_{1}\right)=\left(u-c v^{n}, v\right)$. So we can choose $c \in \mathbb{C}^{\times}$in such a way that $\operatorname{deg} u_{1}<\operatorname{deg} u$. We can continue this procedure recursively until we reach one of the pairs $\left(u_{s}, v_{s}\right)=(\alpha t, 0)$ or $\left(u_{s}, v_{s}\right)=(0, \beta t)$, where $\alpha, \beta \in \mathbb{C}^{\times}$. Then the product $\varphi=\varphi_{s} \circ \ldots \circ \varphi_{1}$ is a required automorphism.

Lemma 4.9. For any $\varphi \in \mathcal{N}_{d, e}$ we have $\varphi(\overline{0})=\overline{0}$ and

$$
G_{d, e} \subseteq \operatorname{Stab}\left(\varphi\left(C_{x}\right)\right) \cap \operatorname{Stab}\left(\varphi\left(C_{y}\right)\right) .
$$

In particular,

$$
\mathcal{N}_{d, e} \cap \operatorname{Aff}\left(\mathbb{A}^{2}\right)=N_{d, e} .
$$

Proof. Since $\varphi$ normalizes the subgroup $G_{d, e}=\langle g\rangle$ we have $\varphi^{-1} \circ g \circ \varphi=g^{k}$ for some $k \in \mathbb{N}$ and so $g \circ \varphi=\varphi \circ g^{k}$. Hence $g\left(\varphi\left(C_{x}\right)\right)=\varphi\left(g^{k}\left(C_{x}\right)\right)=\varphi\left(C_{x}\right)$ and, similarly, $g\left(\varphi\left(C_{y}\right)\right)=\varphi\left(C_{y}\right)$. This yields (25). From

$$
g(\varphi(\overline{0}))=\varphi\left(g^{k}(\overline{0})\right)=\varphi(\overline{0})
$$

we deduce that $\varphi(\overline{0}) \in\left(\mathbb{A}^{2}\right)^{g}=\{\overline{0}\}$ i.e. $\varphi(\overline{0})=\overline{0}$. Now the last assertion follows easily.

Lemma 4.10. If $e^{2} \not \equiv 1 \bmod d$ then

$$
\mathcal{N}_{d, e}=\left\langle\mathcal{N}_{d, e}^{+}, \mathcal{N}_{d, e}^{-}\right\rangle,
$$

while for $e^{2} \equiv 1 \bmod d$,

$$
\mathcal{N}_{d, e}=\left\langle\mathcal{N}_{d, e}^{+}, N_{d, e}\right\rangle=\left\langle\mathcal{N}_{d, e}^{+}, \tau\right\rangle .
$$


Proof. For $\varphi \in \mathcal{N}_{d, e}$ we let $C=\varphi^{-1}\left(C_{y}\right)$. By lemma 4.9 (see (25)) the cyclic group $G_{d, e}$ stabilizes $C$ and $\overline{0} \in C$. By virtue of lemma 4.8 there is an automorphism $\psi \in\left\langle\mathcal{N}_{d, e}^{+}, \mathcal{N}_{d, e}^{-}\right\rangle$ which sends $C$ to one of the coordinate axes $C_{x}, C_{y}$. Letting $\gamma=\psi \circ \varphi^{-1} \in \mathcal{N}_{d, e}$ we get $\gamma\left(C_{y}\right)=\psi(C) \in\left\{C_{x}, C_{y}\right\}$. If $\gamma\left(C_{y}\right)=C_{x}$ then the images of the coordinate axes $\pi\left(C_{x}\right)$ and $\pi\left(C_{y}\right)$ are equivalent in the surface $X_{d, e}$. According to theorem 3.5 in this case $e^{2} \equiv 1 \bmod d$ and $\tau \in N_{d, e}$.

Thus $\gamma\left(C_{y}\right)=C_{y}$ (i.e. $\left.\gamma \in \operatorname{Stab}\left(C_{y}\right)\right)$ if $e^{2} \not \equiv 1 \bmod d$. By proposition 2.8 in this case

$$
\operatorname{Stab}\left(C_{y}\right) \cap \mathcal{N}_{d, e}=\operatorname{Jonq}^{+}\left(\mathbb{A}^{2}\right) \cap \mathcal{N}_{d, e}=\mathcal{N}_{d, e}^{+} .
$$

Hence $\varphi \in\left\langle\mathcal{N}_{d, e}^{+}, \mathcal{N}_{d, e}^{-}\right\rangle$and so (27) follows.

Assume further that $e^{2} \equiv 1 \bmod d$ and $\gamma\left(C_{y}\right)=C_{x}$. Then $\tau \circ \gamma\left(C_{y}\right)=C_{y}$ and so $\tau \circ \gamma \in \mathcal{N}_{d, e}^{+}$ and $\gamma \in\left\langle\mathcal{N}_{d, e}^{+}, \tau\right\rangle$. It follows that

$$
\varphi=\gamma^{-1} \circ \psi \in\left\langle\mathcal{N}_{d, e}^{+}, \mathcal{N}_{d, e}^{-}, \tau\right\rangle=\left\langle\mathcal{N}_{d, e}^{+}, \tau\right\rangle=\left\langle\mathcal{N}_{d, e}^{+}, N_{d, e}\right\rangle .
$$

Now the proof is completed.

We need the following analog of lemma 4.1 in [27], see also [50, Theorem 5.3.1] and [52, Lemma 1.9]. For the reader's convenience we provide a short argument.

Lemma 4.11. Let as before $1 \leq e<d$, where $\operatorname{gcd}(d, e)=1$. Consider an automorphism $\varphi \in \operatorname{Aut}\left(\mathbb{A}^{2}\right)$ with components $u, v \in \mathbb{C}[x, y]$, written as an alternating product

(29) $\varphi=\varphi_{s} \cdot \ldots \cdot \varphi_{1} \quad$ with $\quad \varphi_{i} \in \mathcal{N}_{d, e}^{ \pm} \backslash N_{d, e}^{ \pm} \quad$ and $\quad \varphi_{i+1} \in \mathcal{N}_{d, e}^{\mp} \backslash N_{d, e}^{\mp}, \quad i=1, \ldots, s-1$, where $s \geq 1$. Then

$$
\operatorname{deg} u>\operatorname{deg} v \quad \text { if } \quad \varphi_{s} \in \mathcal{N}_{d, e}^{+} \quad \text { and } \quad \operatorname{deg} u<\operatorname{deg} v \quad \text { if } \quad \varphi_{s} \in \mathcal{N}_{d, e}^{-} .
$$

In both cases

$$
\operatorname{deg} \varphi:=\max \{\operatorname{deg} u, \operatorname{deg} v\}=\prod_{i=1}^{s} \operatorname{deg} \varphi_{i} .
$$

Proof. Both assertions are evidently true if $s=1$. Letting $s>1$ we assume by induction that they hold for the product $\psi=\varphi_{s-1} \cdot \ldots \cdot \varphi_{1}=(\tilde{u}, \tilde{v})$. Thus

$$
\operatorname{deg} \psi=\prod_{i=1}^{s-1} \operatorname{deg} \varphi_{i}
$$

$\operatorname{deg} \tilde{u}<\operatorname{deg} \tilde{v}$ if $\varphi_{s-1} \in \mathcal{N}_{d, e}^{-}$(i.e. $\varphi_{s} \in \mathcal{N}_{d, e}^{+}$), and $\operatorname{deg} \tilde{u}>\operatorname{deg} \tilde{v}$ otherwise. In the former case the induction step goes as follows (the proof in the latter case is similar). By lemma 4.5(a) we can write $\varphi_{s}$ as in (7), where $f \in A_{d, e}$ and $\operatorname{deg} f \geq 2$. Letting

$$
\varphi:(x, y) \longmapsto(u, v)=(\alpha \tilde{u}+f(\tilde{v}), \beta \tilde{v})
$$

from (31) we obtain

$$
\operatorname{deg} u=\operatorname{deg} f(\tilde{v})=\operatorname{deg} f \cdot \operatorname{deg} \tilde{v}=\operatorname{deg} \varphi_{s} \cdot \operatorname{deg} \psi=\prod_{i=1}^{s} \operatorname{deg} \varphi_{i} \geq 2 \operatorname{deg} \tilde{v}>\operatorname{deg} \tilde{v}=\operatorname{deg} v .
$$

This ends the proof.

Now we can deduce the first part of proposition 4.4. 
Lemma 4.12. If $e>1$ then

$$
\left\langle\mathcal{N}_{d, e}^{+}, \mathcal{N}_{d, e}^{-}\right\rangle \simeq \mathcal{N}_{d, e}^{+} *_{\mathbb{T}} \mathcal{N}_{d, e}^{-}
$$

In particular, if $e^{2} \not \equiv 1 \bmod d$ then

$$
\mathcal{N}_{d, e} \simeq \mathcal{N}_{d, e}^{+} *_{\mathbb{T}} \mathcal{N}_{d, e}^{-} .
$$

Proof. The second assertion follows from the first by virtue of lemma 4.10. To show the first one we recall that for $e>1$

$$
\mathcal{N}_{d, e}^{+} \cap \mathcal{N}_{d, e}^{-}=\mathbb{T}=N_{d, e}^{+}=N_{d, e}^{-},
$$

see (19) and (20). By a standard procedure (see [32, Ch. IX, §35, (6)]) any element $\Phi \in$ $\left\langle\mathcal{N}_{d, e}^{+}, \mathcal{N}_{d, e}^{-}\right\rangle$can be written in the form

$$
\Phi=t \varphi=t \varphi_{s} \cdot \ldots \cdot \varphi_{1},
$$

where $t \in \mathbb{T}, s \geq 0$, and for $s>0$ the factors $\varphi_{i} \notin \mathbb{T}$ are as in (29). If $s>0$ then by (30) we obtain

$$
\operatorname{deg} \Phi=\operatorname{deg} \varphi=\prod_{i=1}^{s} \operatorname{deg} \varphi_{i}>1 .
$$

Hence $\Phi=\mathrm{id}$ if and only if $s=0$ and $t=\mathrm{id}$. Thus there is no non-trivial relation in the group $\left\langle\mathcal{N}_{d, e}^{+}, \mathcal{N}_{d, e}^{-}\right\rangle$between elements of the generating subgroups $\mathcal{N}_{d, e}^{ \pm}$and so (32) holds (cf. $[52, \S 13])$.

To finish the proof of proposition 4.4 we need the following auxiliary result from the combinatorial group theory.

Theorem 4.13. (Hanna Heumann [40, Corollary 8.11]) In the amalgamated free product $G=A *_{C} B$ with the unified subgroup $C=A \cap B$, consider two subgroups $\tilde{A} \subseteq A$ and $\tilde{B} \subseteq B$, and let $\tilde{G}=\langle\tilde{A}, \tilde{B}\rangle$. Assume that $\tilde{A} \cap C=\tilde{C}=\tilde{B} \cap C$. Then $\tilde{G}=\tilde{A} *_{\tilde{C}} \tilde{B}$.

The next lemma proves the second part of proposition 4.4.

Lemma 4.14. For $e^{2} \equiv 1 \bmod d$ we have

$$
\mathcal{N}_{d, e} \simeq \mathcal{N}_{d, e}^{+} *_{N_{d, e}^{+}} N_{d, e} .
$$

In particular, for $e=1$

$$
\mathcal{N}_{d, 1} \simeq \mathcal{N}_{d, 1}^{+} *_{B^{+}} \mathrm{GL}(2, \mathbb{C}), \text { where } B^{+}=\mathcal{N}_{d, e}^{+} \cap \mathrm{GL}(2, \mathbb{C}),
$$

while if $e>1$ and $e^{2} \equiv 1 \bmod d$ then

$$
\mathcal{N}_{d, e} \simeq \mathcal{N}_{d, e}^{+} *_{\mathbb{T}} N(\mathbb{T}), \text { where } \mathbb{T}=\mathcal{N}_{d, e}^{+} \cap N(\mathbb{T}) .
$$

Proof. We assume in the sequel that $e^{2} \equiv 1 \bmod d$. Let us note first that (35) and (36) are formal consequences of (34) since

$$
N_{d, e}=\left\{\begin{array}{ll}
\mathrm{GL}(2, \mathbb{C}) & \text { if } e=1 \\
N(\mathbb{T}) & \text { if } e>1
\end{array} \quad \text { and } \quad N_{d, e}^{+}= \begin{cases}B^{+} & \text {if } e=1 \\
\mathbb{T} & \text { if } e>1 .\end{cases}\right.
$$

Let us show (34). It follows from our definitions and lemma 4.9 that

$$
\mathcal{N}_{d, e}^{+} \cap \operatorname{Aff}^{+}\left(\mathbb{A}^{2}\right)=N_{d, e} \cap \operatorname{Aff}^{+}\left(\mathbb{A}^{2}\right)=N_{d, e}^{+} .
$$


Letting

$$
A=\mathrm{JONQ}^{+}\left(\mathbb{A}^{2}\right), \quad B=\operatorname{Aff}\left(\mathbb{A}^{2}\right), \quad \text { and } \quad C=A \cap B=\operatorname{Aff}^{+}\left(\mathbb{A}^{2}\right)
$$

by the Jung-van der Kulk theorem 1.4 we obtain $G=\operatorname{Aut}\left(\mathbb{A}^{2}\right)=A *_{C} B$. Letting further

$$
\tilde{A}=\mathcal{N}_{d, e}^{+}, \quad \tilde{B}=N_{d, e}, \quad \text { and } \quad \tilde{C}=N_{d, e}^{+}=\tilde{A} \cap \tilde{B}
$$

we see by (37) that $\tilde{A} \cap C=\tilde{B} \cap C=\tilde{C}$. Now (34) follows by applying Hanna Neumann's theorem 4.13 .

4.2. Algebraic groups actions on affine toric surfaces. We can deduce now the following analog of the Kambayashi-Wright theorem 1.6 for affine toric surfaces.

Theorem 4.15. Let $G \subseteq \operatorname{Aut}\left(X_{d, e}\right)$ be an algebraic group acting on an affine toric surface $X_{d, e}$, where as before $1 \leq e<d$ and $\operatorname{gcd}(d, e)=1$.

(a) If $e^{2} \not \equiv 1 \bmod d$ then $G$ is conjugate in the group Aut $\left(X_{d, e}\right)$ to a subgroup of one of the groups $\mathcal{N}_{d, e}^{+} / G_{d, e}$ and $\mathcal{N}_{d, e}^{-} / G_{d, e}$.

(b) If $e^{2} \equiv 1 \bmod d$ and $e>1$ then $G$ is conjugate to a subgroup of one of the groups $\mathcal{N}_{d, e}^{+} / G_{d, e}$ and $N(\mathbb{T}) / G_{d, e}$.

(c) If $e=1$ then $G$ is conjugate to a subgroup of one of the groups $\mathcal{N}_{d, e}^{+} / G_{d, e}$ and $\operatorname{GL}(2, \mathbb{C}) / G_{d, e}$.

Proof. Consider the canonical surjection

$$
\pi_{*}: \mathcal{N}_{d, e} \rightarrow \mathcal{N}_{d, e} / G_{d, e} \simeq \operatorname{Aut}\left(X_{d, e}\right)
$$

(see lemma 4.3). The algebraic group $\tilde{G}=\pi_{*}^{-1}(G)$ has bounded degree. Under the assumption of (a) we can conclude that the length $s=\operatorname{length}(\varphi)$ in (29) is uniformly bounded for all $\varphi \in \tilde{G} \backslash \mathbb{T}$. The same holds for $\pi_{*}(\varphi) \in G$ with respect to the free amalgamated product structure (21) as in theorem 4.2. Now (a) follows by Serre's theorem [46] (cf. subsection 1.2). Due to (34)-(36) a similar argument applies also in the remaining cases (b) and (c).

The following corollary is immediate.

Corollary 4.16. Any connected, unipotent subgroup $U \subseteq \operatorname{Aut}\left(X_{d, e}\right)$ is abelian.

For a reductive group acting on $X_{d, e}$, the following analog of proposition 2.5 holds.

Theorem 4.17. Let $G \subseteq \operatorname{Aut}\left(X_{d, e}\right)$ be a reductive algebraic group.

(a) If $e^{2} \not \equiv 1$ mod $d$ then $G$ is conjugate in the group $\operatorname{Aut}\left(X_{d, e}\right)$ to a subgroup of the torus $\mathbb{T} / G_{d, e}$.

(b) If $e^{2} \equiv 1 \bmod d$ and $e>1$ then $G$ is conjugate to a subgroup of the quotient $N(\mathbb{T}) / G_{d, e}$.

(c) If $e=1$ then $G$ is conjugate to a subgroup of the quotient $\mathrm{GL}(2, \mathbb{C}) / G_{d, e}$.

Proof. Clearly the group $\tilde{G}=\pi_{*}^{-1}(G) \subseteq \mathcal{N}_{d, e}$ is reductive. By theorem 4.15 we may assume that $\tilde{G}$ is a subgroup of one of the corresponding factors. It suffices to restrict to the case where $\tilde{G} \subseteq \mathcal{N}_{d, e}^{ \pm}$, since in the other case the assertions are evidently true. In the former case by proposition 2.5 the group $\tilde{G}$ is abelian and conjugate to a subgroup of the torus $\mathbb{T}$ via an element $\mu \in U^{ \pm}$. We claim that such an element $\mu$ can be chosen within the subgroup $U^{ \pm} \cap \mathcal{N}_{d, e}^{ \pm}$. Let us show this assuming that $\tilde{G} \subseteq \mathcal{N}_{d, e}^{+}$, the other case being similar. Indeed, consider an element $\varphi^{+} \in \tilde{G} \subseteq \mathcal{N}_{d, e}^{+}$as in (7). By lemma 4.5 we have $f=\sum_{m \geq 0} a_{m} y^{m} \in A_{d, e}$, 
that is, $a_{m}=0 \forall m \not \equiv e \bmod d$. Hence in (12) we can also choose $g=\sum_{m>0} b_{m} y^{m} \in \mathbb{k}[y]$ so that $b_{m}=0 \forall m \not \equiv e \bmod d$ and so $g \in A_{d, e}$ too. Again by lemma 4.5 the latter ensures that $\mu \in U^{+} \cap \mathcal{N}_{d, e}^{+}$. Since $\tilde{G}$ is abelian, by virtue of lemma 2.4 such an element $\mu$ can be found simultaneously for all $\varphi^{+} \in \tilde{G}$. Since $\mu$ normalizes the cyclic group $G_{d, e}$ it descends to an automorphism $\bar{\varphi} \in \operatorname{Aut}\left(X_{d, e}\right)$ that conjugates $G$ to a subgroup of the torus $\mathbb{T} / G_{d, e}$. The proof is completed.

The following simple example clarifies case (c) above.

Example 4.18. The surface $X_{d, 1}$ is a Veronese cone i.e., the affine cone over a rational normal curve $\Gamma_{d}$ of degree $d$ in $\mathbb{P}^{d}$. The group $\mathrm{GL}(2, \mathbb{C})$ acts naturally on $X_{d, 1}$ (inducing the action of $\operatorname{PGL}(2, \mathbb{C})$ on $\left.\Gamma_{d}\right)$ via the standard irreducible representation on the space of binary forms of degree $d$.

\section{ACYCLIC CURVES AND AUTOMORPHISM GROUPS OF NON-TORIC QUOTIENT SURFACES}

In this section we classify acyclic curves on the quotient $X=\mathbb{A}^{2} / G$ of the affine plane by a nonabelian finite linear group $G$ and describe the automorphism groups of such surfaces.

We assume in the sequel that the finite group $G \subseteq \operatorname{GL}(n, \mathbb{C})$ is small that is, does not contain any pseudoreflection. Recall that a pseudoreflection on $\mathbb{A}^{n}$ is a non-identical linear transformation of finite order fixing pointwise a hyperplane. By the Chevalley-ShephardTodd Theorem, the quotient space $\mathbb{A}^{n} / G$ of a finite linear group $G \subseteq \operatorname{GL}(n)$ is isomorphic to $\mathbb{A}^{n}$ if and only if $G$ is generated by pseudoreflections. Assuming that this is not the case and considering the normal subgroup $H \triangleleft G$ generated by all pseudoreflections, we can decompose the quotient morphism $\pi: \mathbb{A}^{n} \rightarrow \mathbb{A}^{n} / G$ into a two-step factorization

$$
\mathbb{A}^{n} \rightarrow \mathbb{A}^{n} / H \simeq \mathbb{A}^{n} \rightarrow X=\mathbb{A}^{n} / G \text {. }
$$

For $n=2$ we obtain in this way a presentation $X \simeq \mathbb{A}^{2} /(G / H)$, where the small linear group $G / H$ acts on $\mathbb{A}^{2}$ freely off the origin. Thus we may assume, without loss of generality, that $G$ is small. Under this assumption $X=\mathbb{A}^{2} / G$ is a normal affine surface with a unique singular point $Q=\pi(\overline{0})$, and the quotient morphism $\pi: \mathbb{A}^{2} \rightarrow X$ is unramified outside this point.

It is well known that any finite subgroup of the group $\mathrm{SL}(2, \mathbb{C})$ either is cyclic, or is a binary dihedral (respectively, binary tetrahedral, octahedral, icosahedral) group, see e.g. [12] or [29]. The finite subgroups of $\mathrm{GL}(2, \mathbb{C})$ are cyclic extensions of these groups; we refer to [42] for their description.

For $n=2$ every small abelian group $G$ is conjugate to a cyclic group $G_{d, e}$ and so $X=\mathbb{A}^{2} / G$ is a toric surface. If $G$ is small but nonabelian then $X$ is non-toric. In the next theorem we examine this alternative possibility.

Theorem 5.1. Let $X=\mathbb{A}^{2} / G$, where $G \subseteq \mathrm{GL}_{2}(\mathbb{C})$ is a nonabelian, finite, small group. Then any irreducible acyclic curve $\mathcal{C}$ in $X$ is the image $\pi(L)$ of an affine line $L \subseteq \mathbb{A}^{2}$ passing through the origin.

Proof. Let $\mathcal{C}$ be an irreducible acyclic curve on $X$. Assume first that $G$ does not pass through the singular point $Q=\pi(\overline{0}) \in X$. Arguing as in the proof of theorem 3.2 we get

$$
\pi^{*}(\mathcal{C})=C^{1}+\ldots+C^{d},
$$


where $d=|G|$, the irreducible components $C^{i}$ are disjoint, $\pi: C^{i} \rightarrow \mathcal{C} \simeq \mathbb{A}^{1}$ is an isomorphism for all $i$, and the group $G$ acts transitively on the set of these components. Since $d>1$, in suitable new coordinates $(x, y)$ on $\mathbb{A}^{2}$ the curves $C^{i}$ are given by equations $y=\kappa_{i}$ with $\kappa_{i} \in \mathbb{C}^{\times}$. Any $g \in G$ sends $y$ to $\lambda_{g} y$, where $g \mapsto \lambda_{g}$ defines a character $\psi: G \rightarrow \mathbb{G}_{m}$. The $G$-action on the set of components being simply transitive, $\psi$ is injective and so $G$ must be abelian, contrary to our assumption. This contradiction shows that $Q \in \mathcal{C}$.

Consider the reduced acyclic plane curve $C=\pi^{*}(\mathcal{C}) \subseteq \mathbb{A}^{2}$ passing through the origin. If $C$ were smooth at the origin then the finite linear group $G$ preserving $C$ would preserve also the tangent line to $C$ at the origin. So by Maschke's Theorem $G$ should be abelian, a contradiction. Thus $\overline{0} \in C$ is a singular point.

By corollary 2.16 the stabilizer $\operatorname{Stab}(C) \supseteq G$ is abelian unless $C$ is equivalent to a union of $r \geq 2$ affine lines through the origin. Since $G$ is assumed to be nonabelian, the latter condition holds indeed.

We claim that $C$ is in fact a union of lines, which yields the assertion. Indeed, consider the irreducible decomposition $C=C^{1}+\ldots+C^{r}$, where $r \geq 2$. Notice that every component $C^{i}$ of $C$ has a unique place at infinity. Since for every $j \neq i, C^{j}=g\left(C^{i}\right)$ for some $g \in G$, where $g$ is linear, we have $\operatorname{deg}\left(C^{j}\right)=\operatorname{deg}\left(C^{i}\right)=\delta$. Assume to the contrary that $\delta>1$. Since $G$ is nonabelian, by Maschke's Theorem the tautological representation $G \hookrightarrow \operatorname{GL}(2, \mathbb{C})$ is irreducible. Hence the induced $G$-action on $\mathbb{P}^{1}=\mathbb{P}\left(\mathbb{A}^{2}\right)$ has no fixed point. It follows that for some pair of indices $i \neq j$, the points at infinity of the projective curves $\overline{C^{i}}$ and $\overline{C^{j}}=\overline{g\left(C^{i}\right)}$ are distinct. By Bezout Theorem these curves meet in $\delta^{2}>1$ points in $\mathbb{P}^{2}$. Since all these points are situated in the affine part $\mathbb{A}^{2}$ and the intersection index of $C^{i}$ and $C^{j}$ at the origin equals 1 , these curves must have extra intersection points in the affine part. This contradicts the fact that $C$ is simply connected. This contradiction ends the proof.

The following corollary is immediate. Part (a) is an analog of theorem 3.7.

Corollary 5.2. Under the assumptions of theorem 5.1 the following hold.

(a) The only irreducible acyclic curves in $X$ are the closures of one-dimensional orbits of the $\mathbb{G}_{m}$-action on $X$ by homotheties.

(b) The only simply connected curves in $X$ are the images of finite unions of affine lines through the origin in $\mathbb{A}^{2}$.

Using theorem 5.1 we can deduce the following description of the automorphism group of $X$, and as well an information on the equivalence classes of irreducible acyclic curves in $X$.

Theorem 5.3. Let as before $X=\mathbb{A}^{2} / G$, where $G$ is a nonabelian small finite subgroup of $\mathrm{GL}(2, \mathbb{C})$. Then the following hold.

(a) $\operatorname{Aut}(X) \simeq N(G) / G$, where the normalizer $N(G)$ of $G$ in $\mathrm{GL}(2, \mathbb{C})$ is a finite extension of the one-torus $\mathbb{T}_{1,1}=Z(\mathrm{GL}(2, \mathbb{C}))$. Consequently, Aut $(X)$ is a finite extension of the one-torus $\mathbb{T}_{1,1} / Z(G)$.

(b) Let $\mathcal{S}$ denote the set of all irreducible acyclic curves $\mathcal{C}=\pi(L)$ on $X$. Then the $\operatorname{Aut}(X)$ action on $\mathcal{S}$ has finite orbits, and the orbit space $\mathcal{S} / \operatorname{Aut}(X)$ is a rational curve.

Proof. (a) We claim that every automorphism $\alpha$ of the quotient surface $X=\mathbb{A}^{2} / G$ comes from an automorphism of $\mathbb{A}^{2}$ normalizing the group $G$. Indeed, since $G$ is small, the restriction $\left.\pi\right|_{\mathbb{A}^{2} \backslash\{\overline{0}\}}: \mathbb{A}^{2} \backslash\{\overline{0}\} \rightarrow X \backslash\{Q\}$ is an unramified Galois cover with the Galois group $G$. Since 
the surface $\mathbb{A}^{2} \backslash\{\overline{0}\}$ is simply connected, this is a universal cover. Furthermore, $\alpha \in \operatorname{Aut}(X)$ fixes the singular point $Q$ and induces an automorphism of the smooth locus $X \backslash\{Q\}$. By the monodromy theorem, both compositions $\alpha \circ \pi, \alpha^{-1} \circ \pi: \mathbb{A}^{2} \backslash\{\overline{0}\} \rightarrow X \backslash\{Q\}$ can be lifted to holomorphic maps $\tilde{\alpha}, \widetilde{\alpha^{-1}}: \mathbb{A}^{2} \backslash\{\overline{0}\} \rightarrow \mathbb{A}^{2} \backslash\{\overline{0}\}$ in such a way that $\tilde{\alpha} \circ \overline{\alpha^{-1}}=\operatorname{id}_{\mathbb{A}^{2} \backslash\{\overline{0}\}}$. According to the Hartogs Principle, the holomorphic automorphism $\tilde{\alpha}$ of $\mathbb{A}^{2} \backslash\{\overline{0}\}$ extends to such an automorphism of the whole plane $\mathbb{A}^{2}$. Let us show that $\tilde{\alpha}$ is (bi)regular. The induced homomorphism $\tilde{\alpha}^{*}: \mathbb{C}[x, y] \rightarrow \operatorname{Hol}\left(\mathbb{A}^{2}\right)$ into the algebra of entire holomorphic functions in two variables sends the ring of invariants $\mathbb{C}[x, y]^{G}$ to the polynomial ring $\mathbb{C}[x, y]$. Hence the entire holomorphic functions $f=\tilde{\alpha}^{*}(x)$ and $g=\tilde{\alpha}^{*}(y)$ are integral over $\mathbb{C}[x, y]$. Consequently, they are of polynomial growth and so are polynomials. Since $\tilde{\alpha}$ covers $\alpha$ it belongs to the normalizer $\mathcal{N}(G)$ of $G$ in the group $\operatorname{Aut}\left(\mathbb{A}^{2}\right)$. This proves our claim.

By theorem 5.1 the image $\alpha(\mathcal{C})$ of an irreducible acyclic curve $\mathcal{C}=\pi(L)$ on $X$ is again such a curve. Hence any lift $\tilde{\alpha} \in \operatorname{Aut}\left(\mathbb{A}^{2}\right)$ of $\alpha$ preserves the collection of lines through the origin. Similarly as in the proof of corollary 2.9, it is easily seen that an automorphism with the latter property is linear i.e., $\tilde{\alpha} \in \mathrm{GL}(2, \mathbb{C})$. Hence $\tilde{\alpha} \in N(G)$. So finally $\operatorname{Aut}(X)=N(G) / G$. The proof of the remaining assertions is easy and can be left to the reader.

Remarks 5.4. 1. Let as before $\mathcal{N}(G)$ denote the normalizer of $G$ in the full automorphism group Aut $\left(\mathbb{A}^{2}\right)$. The quotient group $\mathcal{N}(G) / G$ embeds into $\operatorname{Aut}(X)$. By virtue of theorem 5.3 it follows that $\mathcal{N}(G)=N(G)$ for any nonabelian small subgroup $G \subseteq \mathrm{GL}(2, \mathbb{C})$.

2. In contrast, for a toric surface $X_{d, e}=\mathbb{A}^{2} / G_{d, e}$ the group $\operatorname{Aut}\left(X_{d, e}\right)$ acts infinitely transitively on the smooth locus $X_{\text {reg }}$ i.e., $m$-transitively for all $m \geq 1$, see [6]. In particular, the group $\operatorname{Aut}(X)$ is infinite dimensional. The equivalence class of any curve $\mathcal{C}$ on $X$ under the $\operatorname{Aut}(X)$-action is infinite dimensional, too. Indeed, given an arbitrary set $K$ of $m$ distinct points on $X_{\text {reg }}$, due to the $m$-transitivity we can interpolate $K$ by an image of $\mathcal{C}$ under a suitable automorphism (cf. corollary 4.18 in [4]).

Example 5.5. Consider the quaternion group

$$
Q_{8}=\left\{ \pm I_{2}, \pm\left(\begin{array}{cc}
i & 0 \\
0 & -i
\end{array}\right), \pm\left(\begin{array}{cc}
0 & 1 \\
-1 & 0
\end{array}\right), \pm\left(\begin{array}{ll}
0 & i \\
i & 0
\end{array}\right)\right\} \subseteq \mathrm{GL}(2, \mathbb{C})
$$

This is a non-splittable central extension of the Klein four-group $V_{4} \simeq \mathbb{Z} / 2 \mathbb{Z} \times \mathbb{Z} / 2 \mathbb{Z}$ by the center $\left\{ \pm I_{2}\right\}$ of $Q_{8}$. The algebra of invariants $\mathbb{C}[x, y]^{Q_{8}}$ is generated by the homogeneous polynomials

$$
f_{1}=x^{4}+y^{4}, \quad f_{2}=x^{2} y^{2}, \quad \text { and } \quad f_{3}=x^{5} y-x y^{5}
$$

satisfying a relation of degree 12 . The map $X=\mathbb{A}^{2} / G \rightarrow \mathbb{A}^{3}$ defined by these polynomials identifies $X$ with a surface of degree 12 in $\mathbb{A}^{3}$ given by a quasihomogeneous polynomial equation with weights $(2,2,3)$. By theorem 5.1 every irreducible acyclic curve $\mathcal{C}$ on $X$ is the image of an affine line $L$ on $\mathbb{A}^{2}$ passing through the origin. Such a curve $\mathcal{C}$ is smooth if and only if the polynomial $f_{3}$ vanishes on $L$, if and only if $L=\mathbb{C} v$, where $v$ is one of the vectors

$$
(1,0), \quad(0,1), \quad(1,1), \quad(1,-1), \quad(1, i), \quad(1,-i) \text {. }
$$

Thus $\mathrm{C}$ coincides with one of the corresponding affine lines on $X$ passing through the singular point $Q$. In particular, these three lines are the only affine lines on $X$ in $\mathbb{A}^{3}$, and the image of any embedding $\mathbb{A}^{1} \hookrightarrow X$ coincides with one of them. These lines are equivalent under 
the action on $X$ of the automorphism group $\operatorname{Aut}(X)=N\left(Q_{8}\right) / Q_{8}$. Indeed, the vectors (38) belong to the same orbit of the normalizer $N\left(Q_{8}\right) \subseteq \mathrm{GL}(2, \mathbb{C})$.

\section{REFERENCES}

[1] S.S. Abhyankar, T.T. Moh. Embeddings of the line in the plane. J. Reine Angew. Math. 276 (1975), $148-166$.

[2] R.C. Alperin. Homology of the group of automorphisms of $\mathbb{k}[x, y]$. J. Pure Appl. Algebra 15 (1979), 109-115.

[3] I.V. Arzhantsev, U. Derenthal, J. Hausen, and A. Laface. Cox rings. arXiv:1003.4229, see also the authors' webpages.

[4] I.V. Arzhantsev, H. Flenner, S. Kaliman, F. Kutzschebauch, and M. Zaidenberg. Flexible varieties and automorphism groups. arXiv:1011.5375 (2011), 41p.

[5] I.V. Arzhantsev, S.A. Gaifullin. Cox rings, semigroups and automorphisms of affine algebraic varieties. Sbornik Math. 201 (2010), 1-21.

[6] I.V. Arzhantsev, K. Kuyumzhiyan, and M. Zaidenberg. Flag varieties, toric varieties, and suspensions: three instances of infinite transitivity. arXiv:1003.3164 (2010), 25p.

[7] T. Asanuma. Nonlinearizable algebraic group actions on $\mathbb{A}^{n}$. J. Algebra 166 (1994), 72-79.

[8] F. Berchtold, J. Hausen. Demushkin's theorem in codimension one. Math. Z. 244 (2003), no. 4, $697-703$.

[9] A. Bialynicki-Birula. Remarks on the action of an algebraic torus on $k^{n}$. I, Bull. Acad. Polon. Sci. Sér. Sci. Math. Astronom. Pkys. 14 (1966), 177-181; II, ibid. 15 (1967), 123-125.

[10] J. Chadzynski, K.T. Krasinski. On a formula for the geometric degree and Jung theorem. Univers. Iagellonicae Acta Mathem. 28 (1991), 81-84.

[11] D.E. Cohen. A topological proof in group theory. Proc. Cambridge Philos. Soc. 59 (1963), 277-282.

[12] J.H. Conway, D.A. Smith. On Quaternions and Octonions. Natick, Massachusetts: AK Peters, Ltd, 2003.

[13] D.A. Cox. The homogeneous coordinate ring of a toric variety. J. Alg. Geom. 4 (1995), 17-50.

[14] W. Dicks. Automorphisms of the polynomial ring in two variables. Publ. Sec. Math. Univ. Autonoma Barcelona 27 (1983), 155-162.

[15] J. Fernández de Bobadilla. A new geometric proof of Jung's theorem on factorisation of automorphisms of $\mathbb{C}^{2}$. Proc. Amer. Math. Soc. 133 (2005), 15-19.

[16] W. Fulton. Introduction to toric varieties. Annals of Math. Studies 131, Princeton University Press, Princeton, NJ, 1993.

[17] M. Furushima. Finite groups of polynomial automorphisms in $\mathbb{C}^{n}$. Tohoku Math. J. (2) 35 (1983), 415424.

[18] M.H. Gizatullin, V.I. Danilov. Automorphisms of affine surfaces. I. Izv. Akad. Nauk SSSR Ser. Mat. 39 (1975), 523-565.

[19] R.V. Gurjar. A new proof of Suzuki's formula. Proc. Indian Acad. Sci. Math. Sci. 107 (1997), $237-242$.

[20] S.M. Gusein-Zade, I. Luengo, and A. Melle-Hernández. On generating series of classes of equivariant Hilbert schemes of fat points. Mosc. Math. J. 10 (2010), 593-602.

[21] A. Gutwirth. An inequality for certain pencils of plane curves. Proc. Amer. Math. Soc. 12 (1961), 631-638.

[22] A. Gutwirth. The action of an algebraic torus on the affine plane. Trans. Amer. Math. Soc. 105 (1962), 407-414.

[23] J.E. Humphreys. Linear algebraic groups. Springer, 1998.

[24] T. Igarashi. Finite Subgroups of the Automorphism Group of the Affine Plane. Master's thesis, Osaka University, 1977.

[25] H. Jung. Über ganze birationale Transformationen der Ebene. J. Reine Angew. Math. 184 (1942), 161174 .

[26] S. Kaliman, M. Koras, L. Makar-Limanov, and P. Russell. $\mathbf{C}^{*}$-actions on $\mathbf{C}^{3}$ are linearizable. Electron. Res. Announc. Amer. Math. Soc. 3 (1997), 63-71.

[27] T. Kambayashi. On the absence of nontrivial separable forms of the affine plane. J. Algebra 35 (1975), $449-456$.

[28] T. Kambayashi. Automorphism group of a polynomial ring and algebraic group action on an affine space. J. Algebra 60 (1979), 439-451. 
[29] A.I. Kostrikin. Introduction to algebra. Universitext. Springer-Verlag, New York-Berlin, 1982.

[30] H. Kraft. Challenging problems on affine $n$-space. Sminaire Bourbaki, Vol. 1994/95. Astrisque 237 (1996), Exp. No. 802, 5, 295-317.

[31] H. Kraft, G. Schwarz. Finite automorphisms of affine $N$-space. Automorphisms of affine spaces. Curaçao, 1994, 55-66, Kluwer Acad. Publ., Dordrecht, 1995.

[32] A.G. Kurosh. The theory of groups. Vol. 1, 2. Chelsea Publishing Co., New York 1960.

[33] S. Lamy. Une preuve géométrique du théorème de Jung. Enseign. Math. (2) 48 (2002), 291-315.

[34] D. Luna. Slices étales. Bull. Soc. Math. France 33 (1973), 81-105.

[35] L.G. Makar-Limanov. On automorphisms of certain algebras. Candidates Dissertation, Moscow State University, 1970. (In Russian.)

[36] J.H. McKay, S.S. Wang. An elementary proof of the automorphism theorem for the polynomial ring in two variables. J. Pure Appl. Algebra 52 (1988), 91-102.

[37] M. Miyanishi. Open algebraic surfaces. CRM Monograph Series, 12. American Mathematical Society, Providence, RI, 2001.

[38] M. Miyanishi, T. Nomura. Finite group scheme actions on the affine plane. J. Pure Appl. Algebra 71 (1991), 249-264.

[39] M. Nagata. On automorphism group of $k[x, y]$. Kinokuniya Book Store, Tokyo, 1972.

[40] H. Neumann. Generalized free products with amalgamated subgroups. Amer. J. Math. 70 (1948), 590625 .

[41] Nguyen Van Chau. A simple proof of Jungs theorem on polynomial automorphisms of $\mathbb{C}^{2}$. Acta Math. Vietnam. 28 (2003), 209-214.

[42] K.A. Nguyen, M. van der Put, and J. Top. Algebraic subgroups of $\mathrm{GL}_{2}(\mathbb{C})$. Indag. Math. (N.S.) 19 (2008), 287-297.

[43] V.L. Popov. On polynomial automorphisms of affine spaces. Izv. Math. 65 (2001), 569-587.

[44] V.L. Popov, E.B. Vinberg. Invariant Theory. In: Algebraic Geometry IV, A.N. Parshin, I.R. Shafarevich (eds), Berlin, Heidelberg, New York: Springer-Verlag, 1994.

[45] R. Rentschler. Opérations du groupe additif sur le plan affine. C. R. Acad. Sci. Paris Sér. A-B 267 (1968), 384-387.

[46] J.-P. Serre. Trees. Springer Monographs in Mathematics. Springer-Verlag, Berlin, 2003.

[47] M. Suzuki. Propriétés topologiques des polynômes de deux variables complexes, et automorphismes algébriques de l'espace $\mathbb{C}^{2}$. J. Math. Soc. Japan 26 (1974), 241-257.

[48] A. Van den Essen. Polynomial automorphisms and the Jacobian conjecture. Progress in Mathematics, 190. Birkhauser Verlag, Basel, 2000.

[49] W. van der Kulk. On polynomial rings in two variables. Nieuw Arch. Wiskunde (3) 1 (1953), 33-41.

[50] D. Wright. Algebras which resemble symmetric algebras. Ph.D. Thesis, Columbia Univ., New York, 1975.

[51] D. Wright. Abelian subgroups of $\operatorname{Aut}_{k}(k[X, Y])$ and applications to actions on the affine plane. Illinois J. Math. 23 (1979), 579-634.

[52] D. Wright. Polynomial automorphism groups. Polynomial automorphisms and related topics, 119, Publishing House for Science and Technology, Hanoi, 2007.

[53] M.G. Zaidenberg. Rational actions of the group $\mathbb{C}^{*}$ on $\mathbb{C}^{2}$, their quasi-invariants and algebraic curves in $\mathbb{C}^{2}$ with Euler characteristic 1. Soviet Math. Dokl. 31 (1985), 57-60.

[54] M.G. Zaidenberg. Affine lines on $\mathbb{Q}$-homology plans and group actions. Transform. Groups 11 (2006), $725-735$.

[55] M.G. Zaidenberg, V.Y. Lin. An irreducible simply connected algebraic curve in $\mathbb{A}^{2}$ is equivalent to a quasi-homogeneous curve. Soviet Math. Dokl. 28 (1983), 200-204.

Department of Algebra, Faculty of Mechanics and Mathematics, Lomonosov Moscow State University, Leninskie Gory 1, Moscow, 119991, Russia

E-mail address: arjantse@mccme.ru

Université Grenoble I, Institut Fourier, UMR 5582 CNRS-UJF, BP 74, 38402 St. Martin D'HÈres CÉdex, France

E-mail address: Mikhail.Zaidenberg@ujf-grenoble.fr 